\title{
„Du kannst doch MEHR!““ - Studierfähigkeit als Bewertungskriterium im Hochschulzugang: Validierungsverfahren zwischen subjektiver Deutung und hochschulischem Gatekeeping
}

\author{
Silke Schreiber-Barsch • Hanna Gundlach
}

Eingegangen: 8. Juni 2018 / Angenommen: 23. Januar 2019 / Online publiziert: 7. Februar 2019

(C) Der/die Autor(en) 2019

Zusammenfassung Der Beitrag diskutiert Validierungsverfahren zum Hochschulzugang auf der Grundlage beruflicher Qualifikation. Dieses Spannungsfeld wird anhand des Eingangsprüfungsverfahrens ( $\$ 38 \mathrm{HmbHG})$ der Universität Hamburg bearbeitet, in dem die Studierfähigkeit beruflich Qualifizierter bewertet wird. Von Interesse ist die qualitative Rekonstruktion von subjektiven Bildungsentscheidungsprozessen in temporalen Übergangsperioden. Hierzu werden Ergebnisse einer Erhebung mit beruflich qualifizierten Studierenden vorgestellt, die sich methodologisch an Grounded Theory orientiert und eine Methodentriangulation nutzt (Teilnehmende Beobachtungen; Gruppendiskussionen; quantitative Fragebogenerhebung). Es zeigt sich, dass das Validierungsverfahren als legitimes und praktikables Instrument zur Messung von Studierfähigkeit angesehen und gleichermaßen in seiner Reproduktionsfunktion diskursiver Ordnungen in Übergängen im Lebenslauf evident wird.

Schlüsselwörter Dritter Bildungsweg · Hochschulzugang · Validierung · Lernergebnis · Studierfähigkeit

\section{"You are capable of MORE!"-Validation procedures to authorise the ability to study between student's perceptions and higher education's gatekeeping}

\footnotetext{
Abstract The paper tackles the issue of procedures for the validation of competences, which are used in the tertiary education sector in order to evaluate an

Jun.-Prof. Dr. S. Schreiber-Barsch $(\bowtie) \cdot$ H. Gundlach, M.A.

Fakultät für Erziehungswissenschaft, Fb. Berufliche Bildung und Lebenslanges Lernen, Universität

Hamburg, 20146 Hamburg, Deutschland

E-Mail: silke.schreiber-barsch@uni-hamburg.de

H. Gundlach, M.A.

E-Mail: hanna.gundlach@uni-hamburg.de
} 
individual's study ability on the basis of his/her vocational qualifications. This contested terrain will be explored by means of the University of Hamburg's validation procedure (the so-called Eingangsprïfungsverfahren, $\$ 38 \mathrm{HmbHG}$ ). The focus of interest will be put on a qualitative reconstruction of individual decision-making processes during these periods of transition. For this aim, the paper introduces findings of a qualitative research project on students with such vocational qualifications background, using Grounded Theory Methodology and a triangulation of methods (participatory observations; focus groups; a quantitative questionnaire). The findings show that the validation procedure is indeed perceived by the sample as a legitimate and feasible instrument for assessing an individual's ability of study—and serves, however, as an instrument for (re)producing discursive orderings of society in periods of transition during the life course.

Keywords Third educational route - Access to tertiary education - Validation · Learning outcome $\cdot$ Study ability

\section{Einleitung}

Als ein strategisches Instrument im System des Lebenslangen Lernens wird Validierung auf europapolitischer Ebene als ein Verfahren gefasst, „,bei dem eine zugelassene Stelle bestätigt, dass eine Person die anhand eines relevanten Standards gemessenen Lernergebnisse erzielt hat" (Rat der EU 2012, Anhang (i)). Im Beitrag ist eine solchermaßen autorisierte Institution eine Hochschule. Sie trifft eine Bewertung darüber, ob bei Studieninteressierten mit beruflicher Qualifikation, aber ohne schulische Hochschulzugangsberechtigung (HZB) und ohne eine Fortbildungsprüfung (Bsp. Meisterprüfung), die sog. „Studierfähigkeit“ (als Lernergebnis) erfüllt ist - und damit eine Zulassung zum Pool der Bewerbungen für einen Studienplatz gewährt werden kann. Die Bewertung setzt als relevanten Standard das Abitur und misst diesen über das Kriterium „Studierfähigkeit“, das für den Validierungsprozess in spezifischer Weise operationalisiert wird (UHH 2014). Studierfähigkeit gilt damit als ein vorgängig zu überprüfendes (und überprüfbares) Lernergebnis.

Die wissenschaftliche Auseinandersetzung um Validierung wird mithin im Kontext Hochschule diskutiert. Empirische Daten einer qualitativen Erhebung mit beruflich qualifizierten Studierenden (den sog. §38-Studierenden) an der Universität Hamburg rücken die Subjektperspektive auf Zugangswege zur Hochschule in den Vordergrund. Von Interesse ist die qualitative Rekonstruktion von subjektiven Bildungsentscheidungsprozessen in temporalen Übergangsperioden, die die bildungswissenschaftliche Übergangs- und Transitionsforschung als ,Zonen der Ungewissheit und Verwundbarkeit" (Walther und Stauber 2013, S. 30) markiert. Fragestellung der Untersuchung ist, inwiefern die hochschulisch-institutionellen Gatekeeping-Prozesse des Hochschulzugangs über $\$ 38 \mathrm{HmbHG}$ von Studienbewerberinnen und -bewerbern der Universität Hamburg als blockierend bzw. unterstützend für Bildungsentscheidungsprozesse zum Ziele des Hochschulzugangs bewertet werden. Unterstellt werden insofern potenzielle Brechungen zwischen hochschulischer und subjektgelagerter Bewertungslogik im Kontext des Validierungsprozesses. Jene Brechungen 
reflektieren und erlauben Rückschlüsse auf das unterliegende Spannungsgefüge: bestehend aus der strukturellen Ebene eines verfassten Übergangssystems im Bildungsbereich am Beispiel der Übergangspolitik der Organisation Hochschule (exekutiert von hochschulischen Gatekeepern), aus diskursiven Ordnungen gesellschaftlicher Normalitätsvorstellungen zu lebenslaufbezogenen Übergängen sowie aus subjektivbiographischen Entscheidungen und Handlungen in Übergängen, wie Struck (2001) und Walther (2015) differenziert haben.

Ziel des Beitrages ist es, zu der sich erst langsam entfaltenden Diskussion um Fragen des Dritten Bildungsweges zur Hochschule (Freitag 2010; HBV 2011; Elsholz 2015; Wolter et al. 2016) und zu sog. nicht-traditionellen Studierenden bzw. second chance learners (Slowey und Schuetze 2012) mit einer handlungstheoretischen Rückbindung an die erziehungswissenschaftliche Übergangs- und Transitionsforschung beizutragen. Dies verspricht, sowohl die wenigen vorliegenden qualitativen Forschungsergebnisse zu dieser Thematik weiter auszudifferenzieren als auch einen Anschluss an die gegenwärtige Debatte um Validierung zu eröffnen (wie u.a. Kil 2016 und Schlögl 2017 in Bezug auf Österreich).

\section{Alternative Zugangswege zur Hochschule: Rahmungen, theoretische Zugänge und Forschungsstand}

Für das Ziel einer qualitativen Rekonstruktion von subjektiven Bildungsentscheidungsprozessen in temporalen Übergangsperioden sind zunächst die wesentlichen bildungspolitischen, hochschulischen wie auch konzeptionellen Rahmungen darzulegen und zwar entlang Ausführungen zum Übergangssystem des Bildungsbereiches (Abschn. 2.1), zu diskursiven Ordnungen des Lernens im Lebenslauf (Abschn. 2.2) sowie zur subjektiv-biographischen Ebene (Abschn. 2.3). Im Verständnis von „Übergängen“ schließen wir an Walther und Stauber (2013) an, die „,die Interaktion zwischen veränderten externen Handlungsanforderungen und Rollenerwartungen an und sich wandelnden Selbstkonzepten des Subjektes“ (ebd., S. 29) als zentral hervorheben; Übergänge stellen derart „Kristallisationspunkte gesellschaftlicher Reproduktion" (ebd.) dar, die wir insbesondere aus der Perspektive des Hochschulbereiches im System des Lebenslangen Lernens betrachten.

\section{1 Übergangssystem Bildungsbereich und Übergangspolitik von Hochschule}

Die wissenschaftlichen wie bildungspolitischen Debatten um alternative Zugangswege zur Hochschule sind zu verorten in den Restrukturierungen des Europäischen Hochschulraums seit Ende der 1990er Jahre im Zuge des BOLOGNA-Prozesses. Sie sollen sicherstellen, das Potenzial der europäischen Bevölkerung im Wettbewerb des internationalen wie globalen Bildungsraumes auszuschöpfen - ,no talent should be left behind" (European Commission et al. 2012, S. 83). In der aktuellen ET2020Strategie der Europäischen Union (EU) („Allgemeine und berufliche Bildung 2020“) findet sich infolgedessen u. a. das Benchmark, wonach bis 2020 mindestens $40 \%$ der 30- bis 34-Jährigen der EU-Mitgliedsstaaten einen Hochschulabschluss besitzen sollen (Eurostat 2018). 
In der Folge sind sowohl auf der EU-Ebene als auch auf nationalen Ebenen bildungspolitische Maßnahmen installiert worden, die nicht nur auf eine rein quantitative Erhöhung von Teilnahme zielen, sondern auch auf eine qualitative Ausweitung der Heterogenität der Studierendenschaft (Orr und Hovdhaugen 2014). Dies soll gelingen über veränderte Zugangsstrukturen zu Hochschulen, sowohl auf der Basis formalrechtlicher Regulierungen als auch über eine Ausweitung der hochschulischen Beratungs- und Unterstützungsleistungen für Studieninteressierte. Der Kultusministerkonferenz-Beschluss (KMK 2009) zum „Hochschulzugang für beruflich qualifizierte Bewerber ohne schulische Hochschulzugangsberechtigung“ (HZB) eröffnete zudem im deutschen Kontext Möglichkeiten zur Aufnahme eines Studiums ohne Abitur. Leitend ist die Unterscheidung zwischen einer allgemeinen HZB auf der Grundlage einer beruflichen Fortbildungsprüfung (Bsp. Meisterprüfung) und einer (oftmals fachgebundenen) HZB für diejenigen, die eine berufliche Ausbildung absolviert, mindestens zwei- bis dreijährige Berufserfahrung gesammelt sowie ein Eignungsfeststellungsverfahren erfolgreich absolviert haben (ggf. ersetzt durch ein mindestens einjähriges Probestudium). Der KMK-Beschluss erlaubt überdies landesspezifische Regulierungen der HZB (ebd.) (s. Abschn. 3.1).

Dies trifft auf die gegenwärtige Agenda-Setzung zur Förderung von Validierung nicht formalen und informellen Lernens. Die EU-Ratsempfehlung von 2012 (Rat der EU 2012) sieht vor, bis 2018 verbindliche Regelungen zur Validierung nicht formal und informell erworbener Kompetenzen, bestehend aus den Schritten Identifizierung, Dokumentierung, Bewertung und Zertifizierung, in den EU-Mitgliedsstaaten einzuführen. Eine solche Validierung von Lernergebnissen soll, so die Argumentation, Beschäftigungsfähigkeit und Mobilität befördern und ,insbesondere sozio-ökonomisch benachteiligte oder niedrigqualifizierte Menschen verstärkt für lebenslanges Lernen motivieren“ (ebd., (1)). Hierzu zählt auch der Zugang in das Hochschulsystem für sog. nicht-traditionelle Studierende. Zielsetzung ist, Lernergebnisse aus nicht formalem und informellem Lernen und somit hochschulische wie außerhochschulische Vorleistungen in eine „Währung“ (CEDEFOP 2016, S. 15) eintauschen zu können, die für die Fortsetzung eines Bildungsweges oder auf dem Arbeitsmarkt Gültigkeit hat (ebd.).

Der Beitrag konkretisiert dies in Bezug auf Zugangsregelungen zu Hochschulen über den sog. Dritten Bildungsweg, d.h. von Studieninteressierten ohne traditionelle HZB (z.B. Abitur). Das deutsche Übergangssystem ist nicht zuletzt durch die Bildungshoheit der Länder gekennzeichnet, die unterschiedliche Übersetzungen der europäischen wie nationalen Regulierungen, teilweise bis hin zur Ebene der einzelnen Hochschule, bedingen (KMK 2009, 2014). Insofern ist das untersuchte Beispiel der Universität Hamburg ein grundsätzlich vergleichbares, trotzdem spezifisches, welches durch die dortige Konstruktion des Validierungsverfahrens einen hohen Erkenntnisgewinn für die Fragestellung verspricht. Das dort installierte hochschulischinstitutionelle Gatekeeping-System des Hochschulzugangs setzt sich zusammen aus rechtlichen Grundlagen (Hamburgisches Hochschulgesetz, HmbHG; HmbHG 2014), aus Verfahren (u. a. Validierungsverfahren der Eingangsprüfung nach §38 HmbHG) und aus Personen, sog. Gatekeepern - und den Studieninteressierten. Zu den Gatekeepern zählen das Beratungspersonal genauso wie hochschulinterne und -externe Prüfungspersonen, administratives Personal in den Studien- und Prüfungsbüros der 
Fakultäten oder auch Hochschullehrende in ihrer Funktion als sog. Studienfachberatende, zuständig für einen Prüfungsbestandteil der Validierung. Die Gatekeeper zeichnen sich insofern durch Funktionen, Aufgaben und Reichweiten der Entscheidungsbefugnis aus, sie besitzen, laut Struck (2001), die Autorität und die konkrete Vollzugsmacht, Entscheidungen als sachgerecht, verfahrensgerecht und verteilungsgerecht zu legitimieren (ebd., S. 45 f). Damit werden sie zu „Schlüsselpersonen mit Entscheidungsautorität“ über knappe Güter (Hochschulzugang) (ebd., S. 37), „die an den Grenzen gesellschaftlicher Teilräume die Anforderungen zum Durchschreiten dieser Räume durchsetzungsstark und definitionsmächtig repräsentieren“ (ebd.). Übergangspolitiken entscheiden mithin über Chancen, Risiken und Handlungsspielräume in Lernbiographie und Lebensverlauf (ebd., S. 33).

Der Bildungsbereich ist somit ein Übergangssystem in Form einer verfassten ,institutionellen Rahmung von Übergängen“ (ebd., S. 32) und gestaltet, eröffnet und limitiert lebenslaufbezogene Handlungsspielräume für Individuen. In Übergängen werden Anschlüsse genauso wie Brüche (re)produziert - nicht zuletzt entlang der diskursiven Ordnungen der Gesellschaft.

\subsection{Lernen im Lebenslauf als (Re)Produktion diskursiver Ordnungen}

Die Arbeiten von u.a. Walther (2015) zeigen, dass im System des Lebenslangen Lernens die Zuordnung von Qualifikationsaufgaben $\mathrm{zu}$ temporalen Altersphasen wirkmächtige Ein- und Ausschlüsse sowie Etikettierungen (re)produziert, die auf diskursiven Ordnungen der Gesellschaft beruht. Biographische Übergänge werden als zu früh (z. B. Elternschaft, Gundlach und Sylla 2017) oder auch als zu spät (z.B. Altersgrenze des Bundesausbildungsförderungsgesetzes, BAföG) definiert. Letzteres greift insbesondere auch für sog. nicht-traditionelle Studierende, die durchschnittlich älter sind, bei ihnen gleichwohl häufig nicht mehr der Anspruch auf BAföGFörderung greift. Bereits die normative Setzung als nicht-traditionell spiegelt eine gesellschaftliche Normalitätsvorstellung von Studierenden wider. Evident sind durchaus ein eher höheres Alter, Herkunft aus im Hochschulsystem eher unterpräsentierten Gruppen oder auch Vorrang berufsbegleitenden Studierens (z. B. Freitag 2010; Elsholz 2015). Letztlich bleibt es aber eine heterogene Gruppe (genauso wie die der sog. traditionellen Studierenden), Lübben et al. (2015) kritisieren vielmehr eine „Inflation von Abgrenzungskriterien“ (ebd., S. 48).

Diskursive Ordnungen der sozialen Welt des „Sinnuniversums Universität“ (Alheit et al. 2008, S. 584) werden gleichermaßen erkennbar. Hierzu gehört auch ein universitärer Habitus, der historisch-durchgängig ,Symptome für eine latente symbolische Schließung des deutschen Hochschulsystems“ (Alheit 2014, S. 206; Herv. i. Orig.) aufweist, und bspw. über akademische Rituale ein ,meritokratisches Selbstverständnis der Wissenschaft" (Krawietz et al. 2013, S. 652) (re)produziert. Das Humboldt'sche Universitätsmodell beansprucht weiterhin symbolische Gültigkeit, mit der Aufladung des Abiturs als legitimes, zugleich unerlässliches Fundament von Studierfähigkeit. Dies erklärt die beharrliche Ungläubigkeit hochschulischer Öffentlichkeit gegenüber der Validität alternativer Zugangswege (Otto und Kamm 2016; Lenz und Schmitt 2016), auch wenn empirische Daten (NEPS-Datensatz) zur Studierfähigkeit von nicht-traditionellen Studierenden dies nicht stützen können 
(Dahm und Kerst 2016). Die symbolische Aufladung des Abiturs manifestiert sich gleichfalls in den von der Universität Hamburg bereitgestellten Informationen zum Validierungsverfahren des Hochschulzugangs für beruflich Qualifizierte, die in der für die untersuchte Kohorte gültigen Fassung (UHH 2014) sowie in einer leicht aktualisierten Fassung (UHH 2017) vorliegen. Die über den KMK-Beschluss von 2009 (KMK 2009) (s. Abschn. 2.1) ausgesprochene gleichberechtigte Wertschätzung vom beruflichen Ausbildungssystem und Hochschulbildung wird hier zugunsten letzterer entschieden. So wird in der aktualisierten Fassung der Sinnbezug zum relevanten Standard Abitur im ,Ziel der Eingangsprüfung“ expliziert: „Die Bewertung durch die Prüferinnen und Prüfer orientiert sich an dem erwarteten Leistungsstand von Abiturientinnen und Abiturienten“ (UHH 2017, S. 7). Während dieser explizite Satz in der Fassung von 2014 noch fehlt, sind die Kriterien des Ziels des Eingangsprüfungsverfahrens, nämlich die Feststellung von Studierfähigkeit, indes gleichgeblieben. Studierfähigkeit wird operationalisiert anhand folgender Kriterien:

- „Denk- und Urteilsfähigkeit,

- Verständnis für wissenschaftliche Fragestellungen sowie für Strukturen und Zusammenhänge,

- die Fähigkeit, Gedanken mündlich und schriftlich in verständlicher Weise darzulegen sowie

- die für ein erfolgreiches Studium erforderliche Beherrschung der deutschen Sprache in Wort und Schrift" (UHH 2014, S. 6).

Dies soll ein Urteil darüber erlauben, ob „Personen, die eine Hochschulzugangsberechtigung nicht besitzen, aufgrund beruflicher Tätigkeit und Qualifikationen aber erwarten lassen, dass sie zu einem Hochschulstudium befähigt sind“ (ebd., S. 3). Der symbolischen Aufladung entsprechend wird in den Hinweisen zur Prüfungsvorbereitung angemahnt, sich rechtzeitig ,,mit niveauvollen Zeitungen, Zeitschriften und anderen Medien zu beschäftigen“ (ebd., S. 7) - aktuell ist „niveauvoll“ durch den Terminus ,,anspruchsvoll“ ersetzt worden (UHH 2017, S. 11), was die unterlegte Normalitätsvorstellung jedoch nicht verändert.

\subsection{Subjektiv-biographische Entscheidungen und Handlungen}

Gemäß dem Ziel einer qualitativen Rekonstruktion von subjektiven Bildungsentscheidungsprozessen in der temporalen Übergangsperiode eines Validierungsverfahrens ist die Subjektperspektive im vorliegenden Beitrag von zentraler Bedeutung (s. auch Abschn. 2.4); hier bündeln sich übergangssystemische Rahmungen und diskursive Ordnungen in subjektiv-biographischen Entscheidungen und Handlungen. Schäffter und Schicke (2016) ist zuzustimmen, dass in der gegenwärtigen „Transformationsgesellschaft“ die „Validierung von Kompetenzen ein funktionales Erfordernis zur Unterstützung der berufsbiografischen Gestaltung diskontinuierlicher bzw. transitorischer Erwerbsbiografien" ist (ebd., S. 26). Bei dem technischadministrativen Verfahren einer Validierung geht es einerseits um die Sicherstellung von Validität, Reliabilität und auch Autorisierung des Instruments und der erzielten Bewertungsergebnisse; andererseits sollen insbesondere auf nicht-formalem und informellem Wege erworbene Kompetenzen sichtbar gemacht und in eine gültige 
„Währung“ (CEDEFOP 2016, S. 15) eingetauscht werden können. Nur so können solche Validierungsverfahren überhaupt "transitorisch produktiv“" werden und eine „subjektive Selbstermächtigung in riskanten Lebenslagen“ erlauben (Schäffter und Schicke 2016, S. 29). Letzteres setzt, nach Schlögl (2017), eine ,substanzielle Würdigung [auch] außercurricularen Lernens" voraus, welches auf tief verwurzelte Spannungsfelder der Debatte um eine gleichberechtigte Wertschätzung vom beruflichen Ausbildungssystem und Hochschulbildung (s. oben) genauso wie von formal sowie nicht-formal und informell erworbenen Lernergebnissen verweist.

Im vorliegenden Kontext wird als relevanter Standard explizit ein Lernergebnis des formalen Bildungssystems gesetzt (Abitur/Studierfähigkeit). Bei den Optionen der Anrechnung außerhochschulischer Vorleistungen wird eine gewisse Öffnung hin zu nicht-formal und informell erworbenen Lernergebnissen erkennbar, indem bei den Zulassungsvoraussetzungen eine Anrechnung von „Zeiten der Kindererziehung, einer Pflegetätigkeit oder eines Wehr-, Ersatz- oder Freiwilligendienstes" auf die Dauer der nachzuweisenden Berufstätigkeit angeboten wird (UHH 2014, S. 2, 2017, S. 5). Insgesamt allerdings spiegelt das untersuchte Validierungsverfahren eine eher am formalen Bildungssystem orientierte Validierung wider, nicht zuletzt durch den hochselektiven Verfahrensablauf. Dies wirft die Frage auf, inwieweit es zu reflexiv und lernförderlich angelegten Validierungsverfahren (Schäffter und Schicke 2016, S. 29) gezählt werden kann.

\subsection{Theoretische Anschlüsse}

Trotz der Installation formalrechtlicher Zugänge zum Hochschulsystem bleibt die Diagnose einer konstant niedrigen Teilnahmequote von Studierenden des Dritten Bildungsweges im Hochschulsystem evident (Autorengruppe Bildungsberichterstattung 2016, S. 127f), deutschlandweit, ,verfügen ein Prozent aller Studierenden über eine Studienzulassung aufgrund einer beruflichen Qualifikation" (Middendorff et al. 2017, S. 29) (auch international z. B. Watson et al. 2013). Vor diesem Hintergrund ist es These des Beitrages, dass die qualitative Rekonstruktion der subjekt-biographischen Perspektiven wichtige Erkenntnisse liefern kann, um zu Erklärungen für diese Lücke zwischen bildungspolitischer Rhetorik, Verfasstheit des Übergangssystems sowie Nutzung des Angebotes beizutragen.

Leitend hierfür ist eine handlungstheoretische Rückbindung an die erziehungswissenschaftliche Übergangs- und Transitionsforschung unter Fokussierung auf eine erwachsenenpädagogische Perspektive. Hochanschlussfähig sind Arbeiten der Biographieforschung (v. a. Alheit und Dausien 2000; Field et al. 2012; Truschkat 2013; Hof 2013), die handlungstheoretisch den Fokus auf die Frage eines „doing biography“ (Dausien 2004) in Anerkennung von subjektgelagerten Gestaltungspotenzialen und -notwendigkeiten in „Zonen der Ungewissheit und Verwundbarkeit“ (Walther und Stauber 2013, S. 30) setzen. Die Gestaltung von Übergängen im Lebenslauf bezieht sich insofern, so Truschkat (2013), immer auf eine „Wechselseitigkeit von subjektiver Verarbeitung sozialer Strukturen einerseits und die biografische Konstruktion sozialer Strukturen andererseits“ (ebd., S. 47). 
Als sozialer Rahmen (Truschkat) des Übergangs ist im Beitrag die Hochschule zu identifizieren, die einen bereits vorstrukturierten Erfahrungs- und Handlungsraum für die Subjekte absteckt, die Subjekte gleichwohl nicht aus ihrer Gestaltungsverantwortlichkeit entlässt. Mit dem Übergangsphänomen des Zugangs zur Hochschule über den $\$ 38$ HmbHG gerät ein selbstinitiierter Übergang ins Untersuchungsblickfeld, der oftmals auf jahrelangen Entscheidungs- und Abwägungsprozessen beruht (s. Abschn. 4), und einen bewusst herbeigeführten Übergang voraussetzt. Gleichwohl, und dies macht das $\S 38$-Validierungsverfahren im vorliegenden Kontext so interessant, bleibt auch nach der getroffenen Entscheidung für die Bewerbung um die Zulassung zum Validierungsverfahren über die Dauer von rund sechs Monaten sowohl auf Seiten der Hochschule als auch auf Seiten der Subjekte der Ausgang ungewiss. Die Hochschule hat mehrere Selektionsschritte im Verfahren installiert, genauso kann und muss das Subjekt im Zuge dessen weitere Entscheidungen fällen. Die Frage nach Entscheidungen lässt insofern Einblicke zu in Handlungsspielräume im „Verhältnis von Freiheit, Determiniertheit und Kontingenz“ (Dausien 2014, S. 41).

Es greift unserer Ansicht nach eine doppelte Temporalisierung des Übergangs im Zuge des Validierungsverfahrens. Zum einen die hochschulseits erzwungene Temporalisierung der Verfahrensschritte über die Dauer mehrerer Monate, die konkret terminierte Entscheidungssituationen (Dausien 2014, S. 40) setzt. Zum anderen ist dies eingebettet in die Dimension der Lebenszeit (ebd.), die auf einer subjektiven Temporalisierung von Bildungsentscheidungsprozessen im Zuge des Übergangsphänomens beruht. Alheit (2003) betont, dass biographische Konstruktionen ,als biografische Temporalisierung sozialer Strukturen begriffen werden" müssen (ebd., S. 25; Herv. i. Orig.). Dieses Aufeinandertreffen zweier Dynamiken der Temporalisierung eröffnet wichtige Erklärungsansätze für die Erhebung (s. auch Field et al. 2012, S. 79) und korrigiert, im Anschluss an Dausien, situationistische Entscheidungsmodelle einer bloßen Abfolge. Erkennbar wird eine „komplexe, mehrschichtige Zeitgestalt, die durch den Eigensinn der Subjekte im Horizont von Erfahrung und Erwartung konstruiert wird und auf die Verschränkung von objektiven und subjektiven, institutionellen und individuellen Zeitlogiken verweist" (Dausien 2014, S. 56).

\section{Empirisches Forschungsfeld und Design der Erhebung}

Zunächst ist das empirische Forschungsfeld des $\$ 38 \mathrm{HmbHG}$ Zugangsverfahrens zur Universität Hamburg in seiner Spezifität kurz zu umreißen, um die Einbettung des Forschungsdesigns und der erzielten Ergebnisse (Abschn. 4) sicherzustellen.

\subsection{Zugangsverfahren zur Universität Hamburg über \$38 HmbHG}

Seit 1979 besteht in Hamburg die Möglichkeit des Zugangs zu einem universitären Studium auch für beruflich Qualifizierte ohne Abitur; die damalige Hochschule für Wirtschaft und Politik (HWP) wurde 2005 mit der Universität Hamburg als Fachbereich Sozialökonomie der Fakultät für Wirtschafts- und Sozialwissenschaften fusioniert (vgl. Schreiber-Barsch 2014). Wesentlich ist hier die Differenzierung zwischen einem Zugang über einen Studienplatz im Fachbereich Sozialökonomie (mit 
einem weniger komplexen Eingangsprüfungsverfahren); über den $\S 37 \mathrm{HmbHG}$ auf der Grundlage einer bestehenden Fortbildungsprüfung (Bsp. Meisterprüfung) und damit ohne zusätzliches Eingangsprüfungsverfahren; sowie über den $\$ 38 \mathrm{HmbHG}$, der durch das restriktive Eingangsprüfungsverfahren gekennzeichnet ist und deshalb von besonderem Interesse für die Frage von Bildungsentscheidungsprozessen ist. Denn es sind bewusste und beharrliche Entscheidungen unbedingte Voraussetzung, um das Validierungsverfahren zu durchlaufen. Untersuchungen zu beruflich qualifizierten Studierenden des Bachelorstudienganges Sozialökonomie, wie von Brändle und Ordemann (2014) vorgelegt, sind insofern nicht anschlussfähig, da die Rahmenbedingungen des Zugangs deutlich weniger komplex und ergo nicht vergleichbar sind.

Das §38-Eingangsprüfungsverfahren wird mit einer Bewerbungsfrist von vier Wochen (Februar) einmal jährlich eröffnet; die Zulassung erfolgt dann für das jeweils nachfolgende Wintersemester (WS). Als Reaktion auf die durchgängig niedrigen Zulassungsquoten, überdies zu spezifischen Studiengängen, wurde zum WS 2014/15 (und damit für die Untersuchungskohorte) erstmals eine Quotenregelung eingeführt. Es müssen seitdem zwischen 3 und 10\% (je nach Ausschöpfung anderer Quotenregelungen) von allen zu vergebenden Studienplätzen einer Fakultät für Studierende nach $\$ 37$ und $\S 38$ offengehalten werden. Im Ergebnis wurden im WS 2014/15 bspw. erstmals sechs Studienplätze für Psychologie an $\$ 38$-Studierende vergeben.

Das §38-Eingangsprüfungsverfahren ist (bis heute) ein mehrstufiges Verfahren, das drei Selektionspunkte vorsieht.

- Bewerbung zur Zulassung zum Verfahren: Zeugnisse und Zertifikate, schriftlicher Bericht (beruflicher Werdegang und Erfahrungen, Begründung Studiengangswahl), Nachweis einer Studienfachberatung in dem angestrebten Studiengang; Zahlung von 204,50€; $\rightarrow$ Selektion

- Schriftliche Prüfungen: Klausur 1 (Thema aus Berufsfeld bzw. angestrebten Studiengang), Klausur 2 (Thema aus öffentlichen Leben), ebenfalls bewertet wird der schriftliche Bericht (s.oben); $\rightarrow$ Selektion

- Mündliche Prüfung: „Die mündliche Prüfung ist keine Wissensabfrage, sondern hat eher den Charakter eines Gespräches“" (UHH 2014, S. 7). Es soll ermittelt werden, „ob die Bewerberin bzw. der Bewerber nach ihrer bzw. seiner persönlichen Reife sowie Denk- und Urteilsfähigkeit geeignet erscheint, das angestrebte Studium aufzunehmen“ (ebd.). $\rightarrow$ Selektion

Vor diesem Hintergrund ergibt sich für die untersuchte Kohorte der Studienbewerberinnen und -bewerber zum WS 2014/15 folgendes Bild (s. Tab. 1), das zu den Zulassungsfällen vor der Einführung der Quotenregelung in Vergleich gesetzt wird.

Tab. 1 Übersicht Zulassungsfälle Universität Hamburg über §38 HmbHG (WS 2013/14 \& 2014/15) (eigene Darstellung)

\begin{tabular}{lll}
\hline $\begin{array}{l}\text { Zulassungsfälle Universität Hamburg über } \$ 38 \\
\text { HmbHG }\end{array}$ & WS 2013/14 & WS 2014/15 \\
\hline $\begin{array}{l}\text { Personen zugelassen zum Eingangsprüfungsver- } \\
\text { fahren }\end{array}$ & 95 & 93 \\
Anzahl der vergebenen Studienplätze & 45 & 60 \\
\hline
\end{tabular}




\subsection{Forschungsdesign der Erhebung}

Ausgangspunkt der Erhebung war die These, dass mit den Studienbewerberinnen und -bewerbern nach $\$ 38 \mathrm{HmbHG}$ eine Gruppe an Personen vorliegt, die mit dem Validierungsverfahren ein spezifisches Übergangsphänomen repräsentiert. Das Phänomen ist gekennzeichnet durch die Kopplung sehr bewusster subjektiver Bildungsentscheidungsprozesse mit einem hohen biografischen Risiko (Dauer des Eingangsprüfungsverfahrens bei langer Ungewissheit des Ausgangs und, bei erfolgter Studienplatzvergabe, kurzfristiger Veränderungsnotwendigkeiten (z. B. Kündigung des Arbeitsplatzes)). Prospektive Gewinne (Wissenserweiterung, soziale/berufliche Statuserhöhung u.Ä.) sind eng geknüpft an mögliche Verluste (sicherer Arbeitsplatz, finanzielle Einbußen u.Ä.). Das Forschungsinteresse galt weniger der fallweisen Rekonstruktion von längerfristigen Lebensverläufen von §38ern, sondern es interessierte das Übergangsphänomen als solches mit unterstellten Brechungen und Ambivalenzen zwischen hochschulischer und subjektgelagerter Bewerbungslogik im Kontext des Validierungsprozesses. Fragestellung war, inwiefern die hochschulischinstitutionellen Gatekeeping-Prozesse des Hochschulzugangs über $\$ 38 \mathrm{HmbHG}$ von Studienbewerberinnen und -bewerbern der Universität Hamburg als blockierend bzw. unterstützend für Bildungsentscheidungsprozesse zum Ziele des Hochschulzugangs bewertet werden.

In der Folge wurde eine empirische Erhebung an der Universität Hamburg durchgeführt (Ende 2014/Anfang 2015; Schreiber-Barsch; Ute Meyer; siehe Endnote des Artikels). Anzuführen sind folgender Untersuchungsaufbau und Zeitlinie der Erhebungssituation (s. Abb. 1).

Methodologisch war das Ziel, das Validierungsverfahren zu untersuchen und eine Varianz in Bewertungslogiken zu ermitteln, was den primär qualitativen Ansatz begründet. Studierfähigkeit wurde insofern nicht als generelle Herausforderung in der Studieneingangsphase aller Studierender untersucht (z. B. Bosse und Trautwein 2014), sondern als ein bereits vorgängig zu überprüfendes (und überprüfbares) Lernergebnis. Für die Schwerpunktsetzung auf die Subjektperspektive zeigten sich biographieanalytische Theoriezugänge als erkenntnisleitend, jedoch lag das Forschungsinteresse nicht auf der Einzelfallebene von $\S 38$ Studieninteressierten, sondern darin, sie als Kollektivität anzuerkennen (wenngleich weder als Milieu, noch als Generation). Die Arbeit von Mangold (1960) hat sich an dieser Stelle als anschlussfähig erwiesen. Mangold leitet eine solche Kollektivität auf der Grundlage der strukturidentischen Erfahrungsaufschichtungen der Beteiligten (Erleben des Eingangsprüfungsverfahrens) ab. Jene Aufschichtungen ermöglichen es den Beteiligten, ,informelle Gruppenmeinungen“ (ebd., S. 68) zu artikulieren, die über Einzelperspektiven hinausgehen. Als Methodologie wurde der Grounded Theory (im Anschluss an Strauss und Corbin 1996; Strübing 2014) gefolgt.

Das Sampling folgte theoretischen und pragmatischen Gesichtspunkten und dem sog. theoretical sampling (Strauss und Corbin 1996; Strübing 2014), das zwei Schritte der Datenerhebung sukzessive aus dem iterativen Auswertungsprozess der Grounded Theory erfolgen ließ und zu einer Triangulation qualitativer und quantitativer Methoden (Flick 2011) führte. Die Datenauswertung der qualitativen Daten nutzte methodisch den Kodierprozess der Grounded Theory gemäß Strauss und Corbin 


\begin{tabular}{|c|c|c|}
\hline $\begin{array}{c}\text { Zugang zur Hochschule beruflich Qualifizierter: } \\
\text { Eingangsprüfungsverfahren nach } \S 38 \mathrm{HmbHG} \text { an der } \\
\text { Universität Hamburg (WS 2014/15) }\end{array}$ & $\begin{array}{l}\text { Anzahl } \\
\text { Personen } \\
\text { WS } \\
2014 / 15\end{array}$ & Zeitlinie \\
\hline $\begin{array}{l}\text { Forschungszugriff } \\
\text { - Quantitative Erhebung: Fragebogen Studienfachberatende } \\
\text { ( } n=133 \text {; Rücklauf: } n=52 \text { ) } \\
\text { - Qualitative Erhebung: Teilnehmende Beobachtung \& } \\
\text { Gruppendiskussionen }(n=8)\end{array}$ & & $\begin{array}{l}\text { März } 2015 \\
\text { September } 2014\end{array}$ \\
\hline \multicolumn{3}{|l|}{ Studienplatz Hochschule } \\
\hline Zusage Studienplatz & $n=60$ & August 2014 \\
\hline $\begin{array}{l}\text { Zulassung zur Bewerbung } \\
\text { um einen Studienplatz }\end{array}$ & & $\begin{array}{l}\text { Juni 2014: Info } \\
\text { Zulassung \& Beginn } \\
\text { Bewerbungsfrist } \\
\text { Studium }\end{array}$ \\
\hline Mehrmonatiges Eingangsprüfungsverfahren & & Februar - Juni 2014 \\
\hline $\begin{array}{l}\text { Tag } \mathbf{x} \text { : } \\
\text { Einreichung Bewerbung zum Eingangsprüfungsverfahren }\end{array}$ & $\begin{array}{l}\text { Zulassung: } \\
\mathrm{n}=93\end{array}$ & Februar 2014 \\
\hline
\end{tabular}

Abb. 1 Zugang zur Hochschule beruflich Qualifizierter: Forschungszugriff und Zeitlinie (eigene Darstellung)

(1996) und Strübing (2014) (Codierung mithilfe von MAXQDA); die quantitativen Daten wurden über SPSS ausgewertet (Pivot-Tabellen).

Die Datenerhebung erfolgte erstens durch teilnehmende Beobachtungen von zwei eintägigen Workshops zum Thema „Studienstart für Studierende ohne Abitur“ an der Universität Hamburg (September 2014) (Erfassung über handschriftliche Beobachtungsprotokolle). Diese Workshops waren ein freiwilliges Angebot für alle §37und §38-Studienanfängerinnen und -anfänger, die in der Kohorte einen Studienplatz erhalten hatten $(n=60)$, noch vor Semesterbeginn des WS 2014/15. Während der Workshops (Teilnehmende insgesamt $n=34$ ) wurden die $\$ 37$ er und $§ 38$ er kurzzeitig zu unterschiedlichen Formaten zugewiesen, so dass nur mit allen anwesenden §38ern eine Gruppendiskussion (Mangold 1960) geführt werden konnte (je Workshop mit je knapp 40 min Länge; Workshop 1, $n=6$; Workshop 2, $n=2$; digitale Erfassung). Die Impulsfrage lautete: ,Sie beginnen nun bald mit dem Studium - wir möchten an dieser Stelle gerne noch mal einen Schritt zurückgehen zum Eingangsprüfungsverfahren. Wie haben Sie das Eingangsprüfungsverfahren erlebt?" Der iterative Auswertungsprozess dieser qualitativen Erhebung ließ insbesondere die Wirkmächtigkeit der Studienfachberatungen als verkörperte Repräsentanten des hochschulischen Gatekeeping in den Vordergrund treten. Deshalb wurde im März 2015 eine zweite Datenerhebung vorgenommen, eine quantitative Online-Fragebogenerhebung (Vollerhebung; lime-survey) von allen im Eingangsprüfungsverfahren beteiligten Studienfachberatungen bzw. hierfür offiziell ausgewiesenen Ansprechpersonen aus bspw. den Studien- und Prüfungsbüros ( $n=133$; Rücklauf $n=52)$.

Zur Reichweite der Erhebung ist anzumerken, dass eine Verzerrung darin gesehen werden kann, dass nur diejenigen Studieninteressierten befragt wurden, die das Validierungsverfahren erfolgreich abgeschlossen haben; dies nimmt zweifellos Einfluss 
auf die retrospektive Rekonstruktion der Erfahrungsaufschichtungen. Die Erfassung auch abgelehnter Studieninteressierter war vorgesehen, konnte aber aus Datenschutzgründen nicht direkt erfolgen und wurde im Laufe der Datenauswertungen wegen dieser Komplexität schließlich ausgekoppelt. Ebenso wäre denkbar gewesen, zwei Erhebungszeitpunkte (Beginn und Ende des Verfahrens) einzuführen; der Zugang zum Feld war allerdings nur punktuell zum Zeitpunkt der Workshops gegeben. Hier eröffnen sich Forschungsdesiderate; gleichwohl ermöglichen die erzielten Ergebnisse umfangreiche Erkenntnisse auf ein hochschulisches Validierungsverfahren unter dem Kriterium von Studierfähigkeit.

\section{Darstellung der Ergebnisse}

Die Darstellung der Ergebnisse folgt dem Auswertungsprozess, indem zunächst die qualitativ ermittelten Ergebnisse (Abschn. 4.1) und nachfolgend die quantitativen (Abschn. 4.2) angeführt werden.

\subsection{Auswertung qualitative Erhebung: Teilnehmende Beobachtungen und Gruppendiskussionen mit Studienanfängerinnen und Studienanfängern}

Die Ergebnisse der qualitativen Erhebung münden in ein heuristisches Modell, das, in der Erklärung des Übergangsphänomens, Bildungsentscheidungsprozesse beruflich Qualifizierter in temporalen Übergangsperioden von Validierungsverfahren zum Hochschulzugang systematisiert (s. Abb. 2). Es basiert auf den im Zuge der Auswertung ermittelten Kategorien und identifiziert als Kernkategorie die subjektive Inkorporation der hochschulischen Logik von Studierfähigkeit.

Die im Modell aufgezeigten Kategorien beruhen auf bestimmten Eigenschaften und wiederum deren Dimensionalisierungen, die die in den Aussagen ermittelte Varianz der Eigenschaften aufzeigen. Zudem ist zu beachten, dass aufgrund des Ablaufs der Erhebungen in den zwei Workshops die Aussagen aus den Teilnehmenden

\begin{tabular}{|c|c|c|}
\hline \multicolumn{3}{|c|}{$\begin{array}{l}\text { Bildungsentscheidungsprozesse beruflich Qualifizierter in temporalen Übergangsperioden von Validierungsverfahren } \\
\text { zum Hochschulzugang - am Beispiel der Universität Hamburg. }\end{array}$} \\
\hline Kategorien & Eigenschaften & Dimensionen \\
\hline \multirow[b]{2}{*}{$\begin{array}{l}\text { ENTSCHEIDUNGSFINDUNG } \\
\text { FÜR / GEGEN STUDIUM }\end{array}$} & Genese Studienwunsch & Biographische Vollendung - rationale Abwägung von Optionen \\
\hline & $\begin{array}{l}\text { Motive für Fortsetzung / } \\
\text { Wiederaufnahme eines formalen } \\
\text { Lernkontextes }\end{array}$ & Leidenschaft für Lernen - Studieren als Lebensform \\
\hline \multirow{4}{*}{$\begin{array}{l}\text { KOLLEKTIVE } \\
\text { ERFAHRUNGSWERTE } \\
\text { ZUM HOCHSCHULISCHEN } \\
\text { GATE-KEEPING }\end{array}$} & Erleben von Prüfungssituationen & Scham-Angst-Genugtuung \\
\hline & $\begin{array}{l}\text { Wahrnehmung von Gate-Keepern in } \\
\text { ihrer hochschulischen Funktion }\end{array}$ & „Abschrecktaktik" - interessierte positive Haltung \\
\hline & „Verwaltungsakt" des Verfahrens & Überarbeitungsnotwendigkeiten - Test eigener Organisationsfähigkeit \\
\hline & Zeitdauer des Verfahrens & Geduldsprobe - finale Willensprüfung \\
\hline \multicolumn{3}{|c|}{$\begin{array}{l}\text { Entscheidungsprozesse und kollektive Erfahrungswerte kulminieren in der Kernkategorie: } \\
\text { Inkorporation der hochschulischen Logik von „Studierfähigkeit“ }\end{array}$} \\
\hline \multirow[b]{2}{*}{$\begin{array}{l}\text { INKORPORATION } \\
\text { DER HOCHSCHULISCHEN LOGIK } \\
\text { VON STUDIERFÄHIGKEIT }\end{array}$} & Sinnuniversum Universität & Qualitätsmerkmal Universität - Qualitätsmerkmal persönliche Eignung \\
\hline & $\begin{array}{l}\text { Subjektiv-biographische } \\
\text { Handlungsstrategien im } \\
\text { Validierungsverfahren }\end{array}$ & „Dahin gehen, wo die Angst ist" - rationale Abwägung \\
\hline
\end{tabular}

Abb. 2 Bildungsentscheidungsprozesse beruflich Qualifizierter in temporalen Übergangsperioden von Validierungsverfahren zum Hochschulzugang (eigene Darstellung) 
Beobachtungen (TB I; II) von sowohl §37ern und §38ern stammen und die Sättigung der Daten stützen; im Mittelpunkt stehen jedoch die Gruppendiskussionen (GD I; II) mit den nur §38ern. Dies ist gekennzeichnet (gemäß der Zeilennummerierung $(Z)$ der Protokolle bzw. der Sequenzen (SQ) der Gruppendiskussionen (mit der Kennzeichnung der jeweiligen Personen, z. B. P2)).

\subsubsection{Entscheidungsfindung für/gegen ein Studium}

Die Daten zeigen, dass die vorgelagerten Prozesse zur Entscheidungsfindung für/ gegen ein Studium eine komplexe, langjährige Struktur besitzen. Dies heben die $\S 37$ er und $\$ 38$ er in den Teilnehmenden Beobachtungen als auch die Aussagen der $§ 38$ er-Gruppendiskussionen hervor. Erkennbar werden die Mühen der Entscheidungsfindung: „Die letzten 1 1/2 Jahre habe ich darauf hingearbeitet. Der Punkt, die Entscheidung zu treffen, hat mich am meisten gekostet“ (TB.II_Z_17-18) (TB.II_Z_87-89; TB.I_Z_14). Verstärkt werde dies durch Skepsis oder Unwissenheit im sozialen Umfeld (,Wie geht das, Du hast kein Abi und kannst studieren?“ TB.II_Z_32-33; auch TB.I_Z-22-23; GD.I.P3_00:37:53-4) oder auch von Eltern (,Meine Eltern haben gesagt, du hast doch einen tollen Job und jetzt bist du auf Hartz IV-Niveau!“" TB.I_Z_26-27). Ähnliches entstehe durch die Unübersichtlichkeit und die formaljuristische Gestalt der Informationen, was abschreckend wirke und hohes Eigenengagement voraussetze (TB.II_Z_50; GD.II.P1_00:02:347; 00:05:04-8). Das Verfahren wirke ,wie ein Riesenwald, wo ich nicht weif, wohin ich mich bewegen soll, damit ich das bekomme, was ich brauche“ (TB.II_Z_60-61). Ist die Entscheidung getroffen, ist eine direkte Umsetzung aufgrund der einmaligen Zulassung pro Jahr nicht immer möglich: ,Habe die Entscheidung vor 1 Jahr gefasst und dachte, es geht direkt los - scheiße, noch ein halbes Jahr warten“" (TB.II_Z_3738).

Diese vorgelagerten Prozesse zur Entscheidungsfindung zeigen zwei wesentliche Eigenschaften: die Genese des Studienwunsches und Motive für Fortsetzung/ Wiederaufnahme eines formalen Lernkontextes.

\section{Genese des Studienwunsches: Zwischen biographischer Vollendung und ra- tionaler Abwägung vorhandener Optionen und Handlungsspielräume Die} Aussagen lassen erkennen, dass der Studienwunsch oftmals keine spontane Idee, sondern auch eine Art biographische Vollendung darstellen kann: ,hadere schon viele Jahre mit diesem Thema, dass ich die Oberstufe nach der elf abgebrochen hab', dass ich nicht studiert hab'. Das hat mich immer sehr traurig gemacht" (GD.II.P1_00:23:54-6). Nicht immer ist klar, welches Studienfach es genau sein soll, aber die Dringlichkeit des Studienwunsches verschärft sich, auch angesichts des Risikos, ggf. irgendwann nicht mehr genug Elan hierfür zu finden (GD.II.P2_00:10:301) (GD.I.P6_00:23:08-7). Dies kann zu einer „Schlüsselsituation“ kulminieren: ,ich hab' die Assistenz der Lehrerin äh gemacht und wenn die mal nicht da war, hab' ICH halt 'n bisschen was machen DÜRFEN“, was den Wunsch weiter reifen lässt: ,und immer hat mich dieser Gedanke begleitet, das kannst du doch auch, Mensch, jetzt mach doch mal ..." (GD.II.P1_00:25:45-8). Unterstützung aus dem sozialen Kontext, z.B. durch den Partner, spielt eine wichtige Rolle (z.B. GD.II.P1_00:25:59-7). Un- 
ter dem Motiv einer biographischen Vollendung kann es insofern zweitrangig sein, ob über das Studium letztendlich de facto eine andere oder verbesserte berufliche Situation erzielt werden kann (GD.II.P2_00:10:44-9).

Demgegenüber kann der Studienwunsch auch aus einer eher rationalen Abwägung vorhandener Optionen und Handlungsspielräume resultieren. In den Teilnehmenden Beobachtungen ( $\$ 37$ er und $\S 38$ er) wurde hervorgehoben, dass ein Studium Chancen auf dem Arbeitsmarkt und Aufstiegsmöglichkeiten verbessere (TB.I_Z_10; TB.I_Z_12; TB.I_Z_13; TB.II_Z_36-37) und damit auch die finanzielle Situation (TB.I_Z_11), d.h.: ,im Gesamtpaket rechnen sich die Investitionen“ (TB.II_Z_28). So werden konkrete Veränderungsimpulse, wie bspw. ein ,großzügiges Abfindungsangebot" (TB.I_Z_16), genutzt, um die Bewerbung zu realisieren. Diese Form der Genese, so auch die Gruppendiskussionen ( $\$ 38 \mathrm{er}$ ), resultiert weniger aus emotional besetzten biographischen Wegmarken, sondern aus rationalen Abwägungen (, es hat alles sein Für und Wider", GD.I.P3_00:37:04-5) zu bspw. passenden biographischen Zeitfenstern (GD.I.P1_00:37:16-9) oder auch aus eher zufälligen Konstellationen: ,Also ich habe drei Jahre lang (..) äh war ein Lehrer mein Vermieter ... und wir ha'm dann des Öfteren bei einem Kaltgetränk das mal erörtert" (GD.I.P2_00:23:372) (auch: GD.I.P5_00:24:03-1; GD.I.P5_00:24:03-1).

\section{Motive für die Fortsetzung/Wiederaufnahme eines formalen Lernkontextes:} Zwischen Leidenschaft für Lernen und der Idee von Studieren als Lebensform Für die Entscheidungsfindung für/gegen ein Studium sind zugleich Motive wirkmächtig, die begründen, weshalb es zu einer Fortsetzung oder Wiederaufnahme eines formalen Lernkontextes kommen soll. Diese Begründungen lassen sich einordnen auf einer Dimensionalisierung zwischen einer generellen Leidenschaft für Lernen bzw. für einen spezifischen Lerngegenstand sowie der Idee, Studieren im Sinne einer bestimmten Lebensform zu verwirklichen.

Leitend für erstere Motive, so zeigen auch die Teilnehmenden Beobachtungen (§37er und §38er), sind Aussagen, die die Leidenschaft für Lernen, auch im Sinne einer intellektuellen Herausforderung, widerspiegeln: „Es reicht mir nicht, was ich kann und weiß" (TB.II_Z_16) (auch: TB.I_Z_6; TB.I_Z_8). In den Gruppendiskussionen (\$38er) heißt es, dass man sich ,,unterfordert“ fühlte, ,ich wollte einfach wieder lernen" (GD.II.P1_00:24:00-4). Diese Annahme wird auf die Gesamtgruppe übertragen (,es eint einfach dieser Wunsch immer 'was lernen zu wollen“; GD.II.P2_00:24:12-0) und als ein kollektives Charaktermerkmal identifiziert: „,das ist dann so angelegt in einem, das wird dann so weiter gehen“ (GD.II.P2_00:24:293). Eine solche Leidenschaft kann auch auf den spezifischen Lerngegenstand der Studienfachrichtung bezogen werden (GD.II.P2_00:26:40-2). Es begründet, weshalb bspw. der Status Gasthörerschaft als nicht mehr zufriedenstellend angesehen wird (GD.II.P2_00:11:14-0).

Die Aufnahme eines formalen Lernkontextes kann zudem darüber erfolgen, dass die Idee von Studieren als Lebensform angestrebt wird. In den Teilnehmenden Beobachtungen ( $\$ 37$ er und §38er) wird das in Äußerungen erkennbar, man wolle endlich „etwas Sinnvolles tun“ (TB.I_Z_5) bzw. in der Betonung, man habe ,schon immer studieren“"wollen (TB.II_Z_24). In den Gruppendiskussionen (§38er) wird dies weiter ausgeführt: Das Studium wird mit dem Begriff ,Spaß““ (GD.II.P2_00:13:26-0) 
belegt und mit der Idee von Studieren als einer Lebensform der „Zurückgezogenheit" (GD.II.P2_00:13:33-2) und dem Ziel, ,andere Welten nachzuvollziehen“ (ebd.). Dies begründe auch den Wechsel von einem berufsbegleitenden Studium zum Vollzeitstudium, um Studieren im Sinne einer Normalitätsvorstellung (,also studieren, ganz normal“; GD.I.P6_00:38:52-0) zu erleben: ,ich will mich darauf konzentrieren können und (.) ja will das auch (.) pur erleben irgendwie“" (GD.I.P6_00:36:01-2).

\subsubsection{Kollektive Erfahrungswerte des Gatekeeping}

Die Aussagen, sowohl der Teilnehmenden Beobachtungen als auch der Gruppendiskussionen, verweisen auf kollektive Erfahrungswerte gegenüber den hochschulischen Gatekeeping-Elementen. Erstens zeigt sich das Erleben der für das Validierungsverfahren konstitutiven Prüfungssituationen als wesentlich, zweitens die Verkörperung der hochschulischen Übergangspolitik durch einzelne Gatekeeper, drittens die Ambivalenz des „Verwaltungsaktes“ des Validierungsverfahrens sowie viertens die Zeitdauer des Verfahrens. Letztere heben die Relevanz der temporalen Dimension in den subjektiven Bedeutungslagerungen hervor (s. Abschn. 2.4).

\section{Das Erleben von Prüfungssituationen: Zwischen Scham - Angst - Genugtuung}

Das Erleben der Prüfungssituationen hängt in hohem Maße davon ab, wie vorgängige Erwartung und dann realer Ablauf in Einklang gebracht werden können. So kann die Studienfachberatung zu einem schambesetzten Erlebnis werden (,,also ich hab' mich richtig geSCḦ̈MT“; GD.I.P1_00:17:56-1), da die Person erwartete, selbst Fragen stellen zu können, doch: ,es war genau anders herum. Der hat mir FRAGEN gestellt und ich bin im Erdboden versunken“ (ebd._00:17:36-6). Resultat war eine kritische Rückmeldung von der Studienfachberatung an die Person (,argumentieren, dass müssen Sie nochmal LERNEN, das geht so NICHT“ (ebd._00:18:06-8)) und dann bei derselben Frustration (,ich dachte mir so, okay ich bin völlig unfähig und das wird alles nichts" (ebd._00:18:23-4)). Neben Scham werden Angst bzw. Unsicherheit ausgelöst. Als Ursachen werden inhaltlich anspruchsvolle Prüfungen genannt (,es ging also richtig zur Sache“, GD.I.P4_00:27:51-5; ,es wurde wirklich auf'n ZAHN gefühlt“, GD.II.P2_00:18:13-8) oder solche, in denen eigene Kompetenzen (bspw. Fremdsprachen) infrage gestellt wurden (GD.II.P1_00:09:10-6; GD.I.P3_00:09:338). Genauso habe die inhaltliche Offenheit der mündlichen bzw. schriftlichen Prüfungselemente Unsicherheit in der Vorbereitung provoziert, man habe sich ,total verrückt gemacht“ (GD.I.P5_00:13:38-6; GD.I.P1_00:03:43-5) und ,wochenlang nur Zeitung gelesen“ (GD.I.P6_00:04:01-6; auch GD.I.P3_00:04:01-6); gleiches löste fehlende Rückmeldung zu Prüfungsleistungen aus (GD.I.P5_00:10:40-3; GD.I.P6_00:09:02-0; GD.I.P1_00:10:19-0).

Interessant ist, dass zwar Scham, Angst und Unsicherheit diesen Situationen zugesprochen werden, sie jedoch retrospektiv auch umgedeutet werden können. Zum einen über den Vollzug der Prüfungssituation (,,alles [war] ganz einfach und nicht so komplex, wie ich mir das vorgestellt hatte" (GD.I.P1_00:04:00-5) (GD.I.P6_00:04:05-4; ebd._00:27:23-7)). Zum anderen wird das Referenzkriterium des relevanten Standards (Abitur) als Vergleich herangezogen: „es war doch einfacher als gedacht (...). Und im Vergleich, also (..) ich hab' kein Abi und im Ver- 
gleich zu den ähm Abiturienten, (..) ja man überlegt, was müssen die alles tun, für so 'ne Abiprüfung, da mussten wir ja denn relativ wenig tun" (GD.I.P1_00:12:486). Die zunächst negativ besetzten Prüfungssituationen können sich insofern auch retrospektiv positiv überlagern mit einem Gefühl der Genugtuung. Letzteres entstehe aus dem Gefühl der Anerkennung der eigenen Erfahrungen und Kompetenzen (GD.I.P3_00:25:46-9), vor allem aber durch das Meistern des Prüfungsverfahrens als finale Bestätigung. Es habe ,beFLÜGELT zu wissen, du kannst es DOCH auch“ (GD.II.P1_00:03:55-2); ,mein Partner hat fast geweint" und ,Mir war eiskalt“ (ebd._00:22:33-9). Diese Genugtuung münde in „Dankbarkeit“", ,dass ich das überhaupt machen darf" (ebd._00:04:06-6). Eine andere Person berichtet:

mein Sohn stand vor der Tür mit einer kleinen Flasche Sekt und die Freude war unendlich, bei ihm und bei mir. Ich hatte das Gefühl, ich hab' den den Himalaya erklommen. Und das war so eine Ausnahme mal sich SELBER, sich selbst was Gutes zu tun. Also in dem Moment habe ich mir selbst was Gutes getan. Nicht des Sekts wegen, sondern, weil ich mich dieser Situation, mich gestellt habe (GD.II.P2_00:10:18-0).

Und später noch mal: ,ja, der Weg war es wert, genau. Und dann der Tag mit der Prüfung, unvergesslich, ein blauer Himmel, mein Sohn neben der Tür. Ein Traum. Es hätte nur noch in der Tagesschau gefehlt" (ebd._00:22:11-8).

\section{Wahrnehmung von Gatekeepern in ihrer Übergangsfunktion: Zwischen Ab-} schrecktaktik und interessierter/positiver Haltung Während Prüfungssituationen an sich sehr unterschiedlich gedeutet werden, hängt die Einschätzung der Erfahrungswerte im Validierungsverfahren zudem davon ab, wie je konkrete Gatekeeper als Verkörperung der hochschulischen Übergangspolitik in der Ausübung ihrer Funktion wahrgenommen werden. Dies variiere zwischen „Abschrecktaktik“ und einer interessierten/positiven Haltung.

In Bezug auf die Studienfachberatung wird bspw. von der „Abschrecktaktik“ (GD.I.P6_00:19:37-9) berichtet, ,also erstmal so'n bisschen äh NEGATIVES erzählt vom Studiengang also erstmal wirklich viele MÄNGEL“ (ebd.) - was jedoch als Funktion der Studienfachberatung und als letztlich hilfreich für die Person interpretiert wird: ,ich glaub' dafür ist so 'ne Studienberatung ja auch eigentlich zuständig, nochmal zu wissen, ist das jetzt wirklich das, was ich machen will“ (ebd._00:19:532). Ähnlich ambivalent wurde eine andere Studienfachberatung wahrgenommen, bei der das Anliegen eines Eingangsprüfungsverfahrens eine deutliche „ÜBERRASCHUNG““ (GD.II.P2_00:15:16-9) hervorgerufen habe, ,ich hatte das Gefühl, dass es nicht so häufig gemacht wird“" (ebd._00:14:28-1). Die Verantwortlichkeit für die Situation des Nicht-Wissens wird jedoch nicht dem Studienfachberatenden übertragen, sondern es werden andere Erklärungen angeboten und die eigene Beharrlichkeit betont: ,es hängt NUR davon ab, welches Fach das ist. Also wenn man jetzt Kirgisisch nimmt oder so, hat man wahrscheinlich ähnliche Erfahrungen" (ebd._00:14:388) bzw. ,ich will da keinem zu nahe treten, vielleicht liegt es auch an mir? Also ich will auf jeden Fall damit sagen, mich hat KEINER von der Uni abgehalten! “ (ebd._00:15:52-2). 
Mehrheitlich jedoch werden in den persönlichen Kontakten positive Erfahrungen gemacht. Es sei über Risiken informiert (GD.I.P3_00:20:19-1), aber der Studienwunsch letztlich positiv bestärkt worden (GD.I.P4_00:22:26-0; GD.I.P6_00:22:505; GD.II.P2_00:15:34-1). Genauso wurden nützliche Informationen und Wissen angeboten (,Ich ging da mit einem großen Packen Wissen raus“, GD.II.P2_00:12:12-6; auch GD.I.P5_00:28:49-3; GD.I.P6_00:22:13-3; GD.I.P3_00:20:31-8) und es habe eine angenehme Atmosphäre geherrscht (GD.II.P1_00:07:46-2; ebd._00:03:23-1).

\section{„Verwaltungsakt“" der Validierung: Zwischen Überarbeitungsnotwendigkeiten und der Interpretation als Test eigener Organisationsfähigkeit Die Erfahrun-} gen aus den Prüfungssituationen sowie aus den persönlichen Kontakten mit den Gatekeepern werden komplementiert durch Rückmeldungen zur formal-administrativen Ebene des Validierungsverfahrens als ein ,Verwaltungsakt“ (GD.II.P2_00:32: 19-7). Jener wird unterschiedlich interpretiert und es deutet sich eine ähnliche Wendung in der Bewertung an: Es werden Überarbeitungsnotwendigkeiten des Verfahrens formuliert, andererseits erlaubt gerade der Verweis auf die schwierigen Bedingungen auch einen positiven Rückschluss auf die eigene Kompetenz als Test der eigenen Organisationsfähigkeit.

Hinweise auf eine Überarbeitungsnotwendigkeit werden auch in den Teilnehmenden Beobachtungen ( $\$ 37$ er und $§ 38$ er) angesprochen. Angeführt werden v. a. Mängel in der Informationsbereitstellung und zu Anerkennungsfragen (,Man kriegt überall etwas Anderes gesagt“ (TB.I_Z_33; TB.II_Z_33) (ähnlich: TB.I_Z_29; TB.I_Z_73; TB.II_Z_31; TB.II_Z_83-84)). In den Gruppendiskussionen (\$38er) wird ebenfalls fehlende Transparenz und Übersichtlichkeit bemängelt (GD.II.P2_00:35:23-9; GD.I.P2_00:21:52-9; GD.I.P1_00:12:22-1), zudem die Komplexität: „Und das dreifach und das zweifach und hier 'n Stempel und da 'n Stempel“" (GD.II.P2_00:32:582). Dies wird auch als Folge der großen Organisationseinheit mit vielen Gatekeepern und unterschiedlichen Entscheidungsbefugnissen gesehen (GD.I.P2_00:21:037; ebd._00:21:44-0), die durchaus zwischen den Fakultäten variieren können (GD.I.P1_00:18:54-4) und letztlich in hoher Unsicherheit resultieren: sowohl in Bezug auf den Zugang (der Gatekeeper ,sagte halt sofort (.) super sie können studieren (..) ich hab' gesagt, aber sie haben mir doch beim letzten Mal gesagt, ich darf es nicht“; GD.II.P1_00:06:07-4) als auch auf den Ablauf des Verfahrens (,Eine Woche vor der schriftlichen Prüfung habe ich 'n Anruf bekommen, der Professor, der ist gar nicht für sie zuständig, äh, jemand anderes bereitet jetzt ihre Frage vor. Und dann, ja, hatte ich 'was ganz anderes“; GD.I.P2_00:05:14-4).

Auf der anderen Seite würden diese Bedingungen eine besondere Qualität von den Personen einfordern, welches auch als Test der Organisationsfähigkeit interpretiert wird (und damit auch als Teil der finalen Willensprüfung; s. unten). Es geht um Sorgfalt, man dürfe es keinesfalls ,, auf die leichte Schulter nehmen“ (GD.II.P1_00:31:468), sondern man müsse „alles tausendmal kontrollieren“ (ebd._00:31:32-1). Denn im Raum stehe immer ,diese böse Drohung, dann werden Sie nicht bla bla bla“ (GD.II.P2_00:32:16-8). Die Verantwortlichkeit für Sorgfalt wird in der Person selbst gesehen, ,ich habe es auf meine Kappe genommen“ (GD.II.P2_00:32:36-6). 
Zeitdauer des Verfahrens: Zwischen anstrengender Geduldsprobe und einer finalen Willensprüfung Was sich zuvor bereits angekündigt hat, kulminiert schließlich in den Aussagen zur Wirkmächtigkeit der temporalen Dimension: der Zeitdauer des Validierungsverfahrens über mehrere Monate. Die Zeitdauer werde während des Verfahrens als anstrengende Geduldsprobe und Phase von Ungewissheit wahrgenommen, doch kann sich dies im Moment des Bestehens der Eingangsprüfung und in der Retrospektive zu einem Moment der finalen Willensprüfung wenden.

Die Gruppendiskussionen (§38er) schildern eindrücklich die emotionale Belastung durch das mehrmonatige Durchschreiten des Eingangsprüfungsverfahrens. Die zeitlich lange Periode des Wartens und der Aufrechterhaltung der Motivation werde als Belastung empfunden (GD.I.P5_00:13:44-9; GD.II.P1_00:03:00-5), gerade auch weil es stets unter dem Vorbehalt stehe, eine nächste Stufe des Verfahrens ggf. nicht zu erreichen (GD.II.P1_00:07:10-9; GD.I.P3_00:31:01-6; GD.I.P1_00:12:55-2). Dies befördere „Selbstzweifel“ (GD.II.P2_00:16:16-9; ,,will ich das eigentlich? Kann ich das eigentlich?" ebd._00:16:11-0) und Ärgern über verpasste Chancen (,hätt'st $d u$ damals ma' 'n bisschen besser aufgepasst (.) in der Schule“, GD.I.P2_00:36:320; auch GD.I.P3_00:36:48-6). Als besonders schwierig wird die Situation des Wartens bei fortlaufender Berufstätigkeit wahrgenommen, in der erst ganz am Ende des Verfahrens klar wird, ob ein Wechsel in der Berufstätigkeit (und damit bspw. eine Kündigung) initiiert werden muss oder nicht (GD.I.P1_00:32:10-4). Durch diese erzwungene Kurzfristigkeit kann der Wechsel unerfreulich werden (,das war kein schönes Ende (.) sag ich mal", GD.I.P2_00:32:04-5) und die weitere Organisation der Existenzsicherung während des Studiums werde nochmals schwieriger (,kurzfristig dann mal eben so BAföG beantragen“, GD.I.P2_00:32:21-3).

Zugleich wird die Temporalität dieses schwierigen Bedingungsgefüges aber auch als Eröffnung einer persönlichen „Bedenkphase“ (GD.II.P2_00:34:33-8) wahrgenommen, in der eine Phase des Wartens und der Ungewissheit erzwungen werde, aber die Freiwilligkeit der Entscheidung für den Verbleib im Verfahren immer beim Subjekt bleibe (GD.II.P1_00:34:18-8; auch GD.II.P2_00:34:21-8). Die Wartezeit des Validierungsverfahrens knüpfe an die langen Jahre der vorgelagerten Entscheidungsfindung an (GD.II.P2_00:11:52-9), so dass die Warteperiode zu einer Transformation führen könne: „dann kann man sich auch immer MEHR damit anfreunden und es wird immer mehr zu einem selbst, zu dem, was man sich so vorstellt. Es verschmilzt so langsam mit der (.) mit der zukünftigen Identität" (GD.II.P2_00:34:521; auch GD.II.P1_00:34:54-7). Letztlich beweise insofern das Aushalten der Phase des Wartens und der Ungewissheit das Bestehen der finalen Willensprüfung: ,man muss es schon wollen, das durch ... auszuhalten“ (GD.I.P4_00:30:21-9), es sich ,erarbeiten" (GD.II.P2_00:21:55-3) (auch GD.II.P1_00:21:08-9). Das könne keiner schaffen, „der das nur mal so nebenbei macht, weil er gerade Bock d'rauf" habe (ebd._00:30:44-6). Man müsse sich hingegen selbst „,disziplinieren“, sich ,immer wieder aufraffen" über die vielen Monate hinweg und sich nicht ,entmutigen“ lassen „,von den ein paar (.) Antworten, die mir nicht gefallen“ (GD.II.P1_00:21:08-9). Denn dies sei später auch im Studium erforderlich: ,Also da wird dich keiner an die Hand nehmen und sagen, du ich hab' jetzt in der Vorlesung auf dich gewartet" (GD.II.P1_00:21:41-7). 


\subsubsection{Inkorporation der hochschulischen Logik von Studierfähigkeit}

Vor diesem Hintergrund wird in den Aussagen sowohl der Teilnehmenden Beobachtungen ( $\$ 37$ er und §38er) als auch insbesondere in den Gruppendiskussionen (\$38er) eindrücklich erkennbar, dass die Prozesse der Entscheidungsfindung und die kollektiven Erfahrungswerte zum hochschulischen Gatekeeping in eine Kategorie münden, die als Kernkategorie identifiziert wurde: die Inkorporation der hochschulischen Logik von Studierfähigkeit. Jene beruht auf den Eigenschaften des Sinnuniversums Universität (in Anschluss an Alheit et al. 2008, S. 584) sowie der subjektivbiographischen Handlungsstrategien.

\section{Sinnuniversum Universität: Zwischen dem Qualitätsmerkmal von Universität und dem Qualitätsmerkmal einer persönlichen Eignung In der subjektiven} Ausdeutung des Sinnuniversums Universität im Kontext des Validierungsverfahrens zeigen sich Vorstellungen von Universität, die mit der Frage von Studierfähigkeit und der subjektiven Einordnung und Bewertung des Validierungsverfahrens eng verknüpft sind. Als Fazit wird erkennbar: Eine hohe Barriere im Zugang zur Universität zeichne die Qualität von Universität aus - und damit auch die Qualität der persönlichen Eignung, die nicht zuletzt das emotionale Erleben von Genugtuung (s. Abschn. 4.1.2) und der finalen Willensprüfung (s. Abschn. 4.1.2) mit impliziert.

So werden in den Teilnehmenden Beobachtungen ( $\$ 37$ er und §38er) der Universität als Qualitätsmerkmale v.a. die individuelle Bewältigung intellektueller Lernleistungen zugeschrieben, es gehe um „Pauken“ (TB.I_Z_24), um ,richtig lernen lernen“(TB.II.Z_96), um „Umgang mit Prüfungsdruck“ (TB.II_Z_108), sich „über längere Zeit konzentrieren“ können (TB.II_Z_101) oder auch um eine angemessene Organisation (Bsp. Stundenplan) der ,weniger strukturierten Situation “ eines Studiums (TB.I_Z_51) im Vergleich zu der einer Ausbildung (,Sonst war der Tag immer vorgegeben“, TB.II_Z_113). In den Gruppendiskussionen (§38er) verdichtet sich der Anschluss an die diskursive Ordnung des Eingangsprüfungsverfahrens und der dortigen Operationalisierung von Studierfähigkeit. Als Referenz wird Schule gesetzt (GD.I.P3_00:09:33-8; GD.I.P6_00:06:24-0), anstelle derer nunmehr wissenschaftliches Arbeiten und Schreiben gefordert sei (GD.I.P5_00:10:048; GD.I.P6_00:06:01-5; GD.I.P1_00:06:11-4). Und genau darin wird das Differenzierungsmerkmal von Universität gesehen: ,man sollte ja doch schon irgendwie den Unterschied merken. Jetzt ist Uni" (GD.I.P3_00:16:02-6). Hier gehe es nicht um das Überprüfen von Fachinhalten (GD.I.P4_00:06:00-1), sondern: „da wird die Studierfähigkeit getestet" (GD.I.P4_00:06:00-1). Das heißt, die Fähigkeiten des wissenschaftlichen Arbeitens und Schreibens, das strukturierte Angehen von Aufgaben und Fragestellungen (GD.I.P1_00:06:16-2; GD.I.P5_00:13:04-9; GD.I.P3_00:06:45-0; GD.I.P4_00:14:07-2), die nicht auf bestehende Wissensinhalte rekurrieren (,dafür studiert man ja“, GD.I.P5_00:13:25-3; GD.I.P4_00:14:135). Dies verweise auf notwendige personale Eigenschaften (,ist der Mensch überhaupt in der Lage, sich damit auseinanderzusetzen? Kann er sich noch öffnen?", GD.I.P2_00:16:48-2; GD.I.P5_00:13:36-8) - und sei mit einer Frage des Niveaus zu verknüpfen. Es zeigt sich das Aufgreifen der Begrifflichkeit aus der Eingangsprüfungsordnung (s. Abschn. 2.2): ,,also denen ging's darum: weniger Bildzeitung mehr 
Süddeutsche“ (GD.I.P2_00:06:29-9) bzw. „ob man dies’ überhaupt dieses Niveau hat" (GD.I.P3_00:06:45-0).

Die Vorstellungen zur Logik von Studierfähigkeit und zum Qualitätsmerkmal einer Universität kristallisierten sich als Distinktionsmerkmal gegenüber Schule und anderen Bildungsinstitutionen als auch gegenüber gescheiterten Bewerberinnen und Bewerbern weiter heraus, indem retrospektiv die Angemessenheit und Gültigkeit des Verfahrens bestätigt bzw. eine noch höhere Barriere gefordert wird - um die eigene Leistung weiter zu erhöhen bzw. retrospektiv zu bestätigen.

Gefordert wird in den Gruppendiskussionen (\$38er), „strenger [zu] benoten“ (GD.I.P3_00:29:29-8), es ,sollte nicht zu einfach gemacht werden“ (GD.II.P1_00: 33:38-4), sondern ,schon 'n bisschen komplexer“ (GD.I.P1_00:12:22-1), damit es „,nicht irgendwie fast geschenkt ist“ (GD.I.P4_00:29:51-1; GD.I.P6_00:29:40-3). Denn ansonsten, so die Argumentation, „da würd ja jeder mit eins abschneiden“" (GD.I.P1_00:12:22-1) und das Verfahren an ,Wertigkeit“ verlieren (GD.II.P1_00:35: 13-4; ebd._00:21:25-9). Dann müsse man fragen, ob das Bestehen des Eingangsprüfungsverfahrens , ̈̈berhaupt irgendwas aussagt“ (GD.I.P4_00:29:52-9). Die Komplexität des Verfahrens bestätige die Eignung als Person, diese finale Willensprüfung (s. Abschn. 4.1.2) gemeistert zu haben: ,das gehört für mich schon zu Studierfähigkeit schon dazu“ (GD.II.P1_00:21:08-9). Deshalb, so das retrospektive Fazit, wolle man ,im Nachhinein nicht verzichten“ (ebd._00:08:19-1; auch GD.II.P2_00:34:116) auf das Eingangsprüfungsverfahren, denn es habe Erfahrungen erlaubt, bspw. vor hochschulischen Prüfungsgremien (,oh Gott, da werden Professoren vor dir sitzen“, GD.II.P1_00:07:40-5) Rede und Antwort stehen zu können (, ich hab' wirklich antworten können ...“, ebd._00:08:13-7) (GD.II.P2_00:35:04-2). Es wird empfunden als finale Anerkennung von Studierfähigkeit: ,okay, die wissen, wen sie vor sich sitzen haben ((einatmen)), die sagen dir, du könntest es schaffen“"(GD.II.P1_00:08:27-2).

Subjektiv-biographische Handlungsstrategien im Validierungsverfahren: Zwischen der Bearbeitung größerer Lebensthemen (,Dahin gehen, wo die Angst ist $^{6)}$ ) und rationaler Vergewisserung Schließlich zeigen sich subjektiv-biographische Handlungsstrategien im Validierungsverfahren, die über konkrete Kontexte (z.B. Prüfungssituationen) hinausgehen und auf eine grundsätzliche Haltung gegenüber biographischen Übergängen verweisen. Diese Haltung spiegelt sich in den Aussagen zwischen der Bearbeitung größerer Lebensthemen einerseits und Strategien eher rationaler Vergewisserung andererseits.

In den Gruppendiskussionen (\$38er) wird unterstrichen, dass man sich ,,nicht mit einer Sache nur bescheiden " (GD.II.P2_00:24:29-3) wolle, sondern, so eine weitere Person: ,weil ich wusste (.) du kannst doch MEHR“ (GD.II.P1_00:23:54-6), „Du bist doch nicht auf den Kopf gefallen“" (ebd._00:25:52-2). Mit dem Bestehen des Validierungsverfahrens schließe sich insofern der Kreis: ,dahin gehen, wo die Angst ist, das ist so immer so mein Lebensmotto“ (ebd._00:30:54-9). Dies unterscheidet sich von anderen subjektiv-biographischen Handlungsstrategien, die eher auf eine rationale Vergewisserung der eigenen Handlungsfähigkeit im Validierungsverfahren und im anstehenden Studium zielen. So wird auf das Sicherheitsnetz der beruflichen Qualifikation verwiesen (,Ich denke, wenn alles schief geht, du bist immer noch xy [Beruf]“, GD.II.P1_00:24:49-7) oder auch auf einen immer existierenden „Plan B“ 
(GD.II.P2_00:23:06-0), der zu dem Fazit führe: „Mehr als hinfallen kann ich nicht“ (ebd._00:15:36-4).

\subsection{Auswertung quantitative Erhebung: Fragebogenerhebung mit hochschulischen Gatekeepern}

In der qualitativen Erhebung hatte sich die Wirkmächtigkeit des Erlebens und der Erfahrungen mit Personen aus dem hochschulischen Gatekeeping herauskristallisiert, insbesondere in Bezug auf die Studienfachberatungen. Diese stellen einen ersten, prägenden Kontakt zu dem anvisierten Studiengang dar, da der Nachweis über eine Studienfachberatung bereits bei der Bewerbung um die Zulassung zum Validierungsverfahren eingereicht werden muss. Die Gewährung eines solchen Beratungstermins bildet insofern eine konkrete Vollzugsmacht im Gatekeeping ab.

\subsubsection{Design}

Im Frühjahr 2015 wurde ein Online-Fragebogen (lime-survey) an 133 im Studieneingangsprüfungsverfahren beteiligte Studienfachberatungen und weitere offiziell registrierte Ansprechpersonen verschickt (Rücklauf $n=52$ ). Insgesamt umfasste die Umfrage 31 Items: 28 geschlossene Fragen sowie dreimal die Möglichkeit einer schriftlichen Kommentierung. Es wurden diejenigen Studienfachberatenden mit Erfahrung (in den letzten zwei Jahren) zu §38-Studieninteressierten identifiziert; 30 der 52 eingegangenen Fragebögen erfüllten dies und wurden in die Analyse einbezogen.

Die beiden größten Gruppen der Teilnehmenden an der Umfrage stellten die Fakultäten Erziehungswissenschaft $(n=7)$ sowie Mathematik, Informatik, Naturwissenschaft $(n=8)$. Insgesamt kamen zwei Drittel $(n=20)$ der Studienfachberatenden aus dem Kreis der Professorenschaft, ein Drittel $(n=10)$ waren Mitarbeitende der Studien- und Prüfungsbüros. Die Mehrheit der Beratungsgespräche dauere, so die Angaben, im Schnitt $30 \min (n=19)$ bis $45 \min (n=9)$.

\subsubsection{Einschätzung von Studierfähigkeit im Rahmen der Studienfachberatungen}

Die Analyse der Daten konzentrierte sich im Gesamtauswertungsprozess auf die Frage, wie die Studienfachberatenden ihren Beitrag einschätzen zur Bewertung der Studierfähigkeit der Studieninteressierten, welches als zentrale Aufgabe der Studienfachberatung identifiziert ist. Hierzu wurden über die Items diejenigen Kriterien abgefragt, die in der Eingangsprüfungsordnung das Kriterium Studierfähigkeit operationalisieren (s. Abschn. 2.2).

Es zeigt sich, dass mehr als zwei Drittel aller Befragten der Aussage zustimmen, in der Studienfachberatung einen Eindruck über die Denk- und Urteilsfähigkeit des Studieninteressierten zu erhalten (eher $(n=17)$ bzw. völlig $(n=5))$. Diese Bewertung passt zu dem Setting einer Face-to-face-Beratung, in der es um die Studienfachwahl und damit zusammenhängende Faktoren geht. Noch deutlicher, und wiederum zum Beratungssetting stimmig, wird dem Beitrag der Studienfachberatung zur Einschätzung der Fähigkeit der Studieninteressierten, Gedanken in verständlicher Weise mündlich darzulegen, zugestimmt (eher $(n=15)$ bzw. völlig $(n=11)$ ). 
Gänzlich eindeutig wird das Bild bei der Einschätzung über die erforderliche mündliche Beherrschung der deutschen Sprache (völlig $(n=21)$ bzw. eher $(n=8)$ ). Bei diesen Komponenten der operationalisierten Studierfähigkeit wird also der Beitrag der Studienfachberatung zur Einschätzung der Studieninteressierten als insgesamt positiv und stimmig mit dem Beratungssetting beurteilt. Hingegen in Bezug auf die Komponente der Einschätzung der Fähigkeit der Studieninteressierten, wissenschaftliche Fragestellungen, Strukturen und Zusammenhänge zu verstehen, zeigt sich kein einheitliches Bild, die Streuung der Antworten ist insgesamt recht breit. Knapp zwei Drittel stimmen eher $(n=11)$ bzw. gar nicht $(n=8) \mathrm{zu}$, wohingegen ein weiteres Drittel eher $(n=9)$ und völlig $(n=2)$ zustimmt. Dieses im Vergleich abstraktere Operationalisierungs-Item der Studierfähigkeit scheint insofern komplexer in einer Beratungssituation zu ermitteln zu sein.

Deutlich wird das Potenzial des Beratungssettings, neben den fachlich-wissenschaftlichen und intellektuellen Kriterien auf die persönlichen Motive und Lebenssituation der Studieninteressierten eingehen zu können. So befürworteten $90 \%$ der Befragten eher $(n=15)$ bzw. völlig $(n=12)$, durch das Beratungsgespräch einen Eindruck über die Sorgen und Befürchtungen der Studieninteressierten in Bezug auf die Aufnahme eines Studiums erhalten zu können. Gleiches gilt für die persönlichen Gründe für die Bewerbung (knapp $90 \%$ eher $(n=10)$ bzw. völlige $(n=16)$ Zustimmung). Eine ähnliche breite Streuung und damit kein klarer Trend in den Antworten wie bei der Einschätzung der Fähigkeit wissenschaftlicher Fragestellungen zeigt sich bei der Bewertung der fachspezifischen Kenntnisse der Studieninteressierten im Zuge des Beratungsgespräches; je ein gleicher Anteil von über $40 \%$ der Befragten stimmt der Einschätzung der Fachspezifik eher nicht zu $(n=13)$ wie diesem eher zugestimmt wird $(n=13)$. Für diese Bereiche scheinen insofern eher die anderen Prüfungselemente (schriftliche und mündliche Prüfungen) als geeigneter angesehen zu werden.

Auch die Frage zur Transferierbarkeit, d.h. ob aus Sicht der Studienfachberatungen das aus der Berufstätigkeit eingebrachte Praxiswissen im weiteren Verlauf des Studiums nützlich sein könnte, bringt keinen klaren Trend in den Antworten hervor; hier war zudem eine offene Antwortmöglichkeit vorhanden. Die Hälfte der Befragten schätzt die Nützlichkeit des aus der Berufstätigkeit mitgebrachten Praxiswissens als mittelmäßig ein. Je etwa ein Viertel schätzen es als gering $(n=8)$ bzw. als hoch ein $(n=7)$. Die schriftlichen Kommentierungen zeigen, dass eine hohe Nützlichkeitseinschätzung insbesondere durch den Praxistransfer des Gelernten begründet wird. Bei einer mittleren Nützlichkeitseinschätzung wird z. B. auf das nur für Teilbereiche des Studiums transferierbare Erfahrungswissen verwiesen sowie auf die Abhängigkeit der Nützlichkeit vom zuvor erlernten und ausgeübten Beruf. Eine geringe Nützlichkeitseinschätzung wird u.a. durch das andere Anforderungsprofil eines wissenschaftlichen Studiums im Vergleich zu praktischen Berufserfahrungen begründet. 


\subsubsection{Hochschulstrategische Überlegungen zur Gestaltung des Zugangs zur Hochschule}

Zum Abschluss des Fragebogens wurden allgemeine Einschätzungen zum Zugang zum Hochschulstudium durch beruflich Qualifizierte abgefragt. Hierbei zeigen sich einige hochschulstrategisch interessante Ergebnisse. Zum einen zeigte sich ein eindeutiger Zuspruch zur Beibehaltung des Eingangsprüfungsverfahrens: 25 der $30 \mathrm{Be}-$ fragten lehnten die Abschaffung des Verfahrens und den Ersatz dieses Verfahrens durch z.B. ein Probestudium ab. Bei der Nachfrage nach der Beurteilung eines Probestudiums herrschte Uneinigkeit. Je etwa die Hälfte sprach sich für $(n=14)$ bzw. gegen $(n=16)$ die Einführung eines Probestudiums aus. Eine Verschärfung des Gatekeepings in Form des Eingangsprüfungsverfahrens wird allerdings eindeutig abgelehnt $(80 \%$ der Befragten $(n=24))$. Insgesamt wird insofern von den Studienfachberatungen für die Erhaltung des Status quo plädiert.

\section{Abschließendes Fazit und Perspektiven}

Abschließend ist zu schlussfolgern, dass sich mit dem heuristischen Modell zur Erklärung des Übergangsphänomens des Eingangsprüfungsverfahrens und durch die Kontrastierung mit der hochschulischen Gatekeeper-Perspektive wesentliche Erkenntnisse für die Diskussion um Validierungsverfahren abzeichnen.

Während für Fragen nach der Validität, Reliabilität und Praktikabilität des Validierungsverfahrens Erkenntnisse sowohl aus Nutzersicht von Studieninteressierten als auch von Repräsentanten des hochschulischen Gatekeepings ermittelt werden konnten, die eine Optimierung des Verfahrens vor allem in der administrativen Prozessierung vorschlagen, aber sich grundsätzlich für die Erhaltung des Status quo aussprechen, pointieren die Ergebnisse der qualitativen Rekonstruktion die Brechungen zwischen hochschulischer und subjektgelagerter Bewertungslogik im Validierungsverfahren. Interessant ist, dass an keiner Stelle in der Datenerhebung von den befragten Subjekten die Angemessenheit oder Legitimität der unterliegenden hochschulischen Logik von Studierfähigkeit oder auch die Gültigkeit des Verfahrens der Überprüfung ihrer Lernergebnisse an sich infrage gestellt wurde. Hier zeigt sich als einflussreiche Dynamik in der retrospektiven Ausdeutung der kollektiven Erfahrungswerte eine temporale Dimension, die in Bezug auf die gleichen Komponenten des Validierungsverfahrens über die Zeitdauer des Verfahrens hinweg eine Umdeutung erkennen lässt. Während so bspw. die Zeitdauer des Verfahrens als eine anstrengende Geduldsprobe wahrgenommen wird - ist sie doch zugleich eine Auszeichnung finaler Willensprüfung. Im gleichen Maße, wie das „Sinnuniversum Universität" die Bildungsentscheidungsprozesse vor und während des Validierungsverfahrens erkennbar verkompliziert, gewährleiste gleichwohl genau dies den Nachweis der Qualität von Universität - und der persönlichen Eignung. Das erfolgreiche Durchlaufen des Eingangsprüfungsverfahrens wird zur persönlichen Abitur-Äquivalenz. 
Ein solches Validierungsverfahren kann insofern, wie mit Schäffter und Schicke (2016) eingangs aufgeworfen, erkennbar „transitorisch produktiv werden“ (ebd., S. 29), dies zeichnen die Ergebnisse eindrücklich nach. Im gleichen Maße evident werden Übergänge in ihrer Charakteristik als „Kristallisationspunkte gesellschaftlicher Reproduktion“ (Walther und Stauber 2013, S. 29), die, und dies führt zu weiteren Forschungsdesideraten, Validierungsinstrumente auch als Instrumente zur Inkorporierung von hochschulischen Gatekeeping-Strukturen erkennbar werden lassen.

Danksagung Wir danken Frau Ute Meyer (UHH) für die Bereitstellung des Feldzuganges und der Unterstützung bei der Erhebung; ebenso danken wir insbesondere Frau Emma Fawcett (HU Berlin) für die Unterstützung in Datenerhebung und Codierung.

Open Access Dieser Artikel wird unter der Creative Commons Namensnennung 4.0 International Lizenz (http://creativecommons.org/licenses/by/4.0/deed.de) veröffentlicht, welche die Nutzung, Vervielfältigung, Bearbeitung, Verbreitung und Wiedergabe in jeglichem Medium und Format erlaubt, sofern Sie den/die ursprünglichen Autor(en) und die Quelle ordnungsgemäß nennen, einen Link zur Creative Commons Lizenz beifügen und angeben, ob Änderungen vorgenommen wurden.

Publisher's Note Springer Nature remains neutral with regard to jurisdictional claims in published maps and institutional affiliations.

\section{Literatur}

Alheit, P. (2003). Biografizität. In R. Bohnsack, W. Marotzki \& M. Meuser (Hrsg.), Hauptbegriffe Qualitativer Sozialforschung (S. 25). Opladen: Leske + Budrich/UTB.

Alheit, P. (2014). Die Exklusionsmacht des universitären Habitus. Exemplarische Studien zur ,neuen deutschen Universität“". In N. Ricken, H-C. Koller \& E. Keiner (Hrsg.), Die Idee der Universität-revisited (S. 195-208). Wiesbaden: Springer VS.

Alheit, P., \& Dausien, B. (2000). Die biographische Konstruktion der Wirklichkeit. Überlegungen zur Biographizität des Sozialen. In E. M. Hoerning (Hrsg.), Biographische Sozialisation (S. 257-283). Stuttgart: Lucius \& Lucius.

Alheit, P., Rheinländer, K., \& Watermann, R. (2008). Zwischen Bildungsaufstieg und Karriere. Studienperspektiven ,nicht-traditioneller Studierender“. Zeitschrift für Erziehungswissenschaft ZfE, 11(4), 577-606.

Autorengruppe Bildungsberichterstattung (2016). Bildung in Deutschland 2016. Bielefeld: W. Bertelsmann.

Bosse, E., \& Trautwein, C. (2014). Individuelle und institutionelle Herausforderungen der Studieneingangsphase. Zeitschrift für Hochschulentwicklung, 9(5), 41-62.

Brändle, T., \& Ordemann, J. (2014). Studierfähigkeit und die Aufnahmeprüfung von Studierenden ohne Abitur am Fachbereich Sozialökonomie der Universität Hamburg. https://www.universitaetskolleg. uni-hamburg.de/de/projekte/tp29/tp29media/braendle-ordemann-2014-studierfaehigkeit-undaufnahmepruefung.pdf. Zugegriffen: 07.06.2018.

CEDEFOP (2016). Europäische Leitlinien für die Validierung nicht formalen und informellen Lernens. Luxemburg: Amt für Veröffentlichungen der Europäischen Union.

Dahm, G., \& Kerst, C. (2016). Erfolgreich studieren ohne Abi? Ein mehrdimensionaler Vergleich des Studienerfolgs von nicht-traditionellen und traditionellen Studierenden. In A. Wolter, U. Banscherus \& C. Kamm (Hrsg.), Zielgruppen Lebenslangen Lernens an Hochschulen (S. 225-263). Münster: Waxmann.

Dausien, B. (2004). Biographieforschung: Theoretische Perspektiven und methodologische Konzepte für eine re-konstruktive Geschlechterforschung. In R. Becker \& B. Kortendiek (Hrsg.), Handbuch Frauen- und Geschlechterforschung. Theorie, Methoden, Empirie (S. 314-325). Wiesbaden: Springer VS.

Dausien, B. (2014). „Bildungsentscheidungen“ im Kontext biografischer Erfahrungen und Erwartungen. Theoretische und empirische Argumente. In I. Miethe, J. Ecarius \& A. Tervooren (Hrsg.), Bildungsentscheidungen im Lebenslauf (S. 39-61). Opladen, Berlin, Toronto: Barbara Budrich. 
Elsholz, U. (Hrsg.). (2015). Beruflich Qualifizierte im Studium: Analysen und Konzepte zum Dritten Bildungsweg. Bielefeld: W. Bertelsmann.

European Commission, EACEA P9, \& Eurydice (2012). The European higher education area in 2012: Bologna Process implementation report, Brussels. https://media.ehea.info/file/2012_Bucharest/79/5/ Bologna_Process_Implementation_Report_607795.pdf. Zugegriffen: 08.01.2018.

EUROSTAT (2018). Bildung und Weiterbildung: EU Benchmarks. http://ec.europa.eu/eurostat/de/web/ education-and-training/eu-benchmarks. Zugegriffen: 24.05.2018.

Field, J., Merrill, B., \& West, L. (2012). Life history approaches to access and retention of non-traditional students in higher education: a cross-European approach. European journal for Research on the Education and Learning of Adults, 3(1), 77-89.

Flick, U. (2011). Triangulation. Eine Einführung (3. Aufl.). Wiesbaden: Springer VS.

Freitag, W. K. (2010). Recognition of prior learning - Anrechnung vorgängig erworbener Kompetenzen: EU Bildungspolitik, Umsetzung in Deutschland und Bedeutung für die soziale und strukturelle Durchlässigkeit zur Hochschule. Düsseldorf: Hans-Böckler-Stiftung.

Gundlach, H., \& Sylla, C. (2017). The challenge of overcoming deficit orientation towards adolescent parents through social research in Germany and in the USA. In J. Schroeder, L. H. Seukwa \& U. Voigtsberger (Hrsg.), Soziale Bildungsarbeit - Europäische Debatten und Projekte (1. Aufl. S. 69-84). Wiesbaden: Springer VS.

HBV (2011). Übergänge. Hessische Blätter für Volksbildung, 3.

HmbHG (2014). Hamburgisches Hochschulgesetz. http://www.landesrecht-hamburg.de/jportal/portal/ page/bshaprod.psml?showdoccase $=1 \&$ st=lr\&doc.id=jlr-HSchulGHArahmen\&doc. part=X\&doc. origin=bs (Erstellt: 18. Juli 2001). Zugegriffen: 07.06.2018.

Hof, C. (2013). Übergänge und Lebenslanges Lernen. In W. Schröer, B. Stauber, A. Walther, L. Böhnisch \& K. Lenz (Hrsg.), Handbuch Übergänge (S. 394-414). Weinheim: Beltz Juventa.

Kil, M. (2016). Individuelle Studierpotenziale wertschätzen: Anerkennung formaler, non-formaler und informell erworbener Kompetenzen (Kompetenzstufe 6 und 7). Der pädagogische Blick, 1, 40-53.

KMK (2009). Hochschulzugang für beruflich qualifizierte Bewerber ohne schulische Hochschulzugangsberechtigung. Beschluss der Kultusministerkonferenz vom 06.03.2008. http://www.kmk.org/ fileadmin/veroeffentlichungen_beschluesse/2009/2009_03_06-Hochschulzugang-erful-qualifizierteBewerber.pdf. Zugegriffen: 07.06.2018.

KMK (2014). Synoptische Darstellung der in den Ländern bestehenden Möglichkeiten des Hochschulzugangs für beruflich qualifizierte Bewerber ohne schulische Hochschulzugangsberechtigung auf der Grundlage hochschulrechtlicher Regelungen. Stand: August 2014. https://www.kmk.org/ fileadmin/veroeffentlichungen_beschluesse/2014/2014_08_00-Synopse-Hochschulzugang-berufl_ Qualifizierter.pdf. Zugegriffen: 07.06.2018.

Krawietz, J., Raithelhuber, E., \& Roman, N. (2013). Übergänge in der Hochschule. In W. Schröer, B. Stauber, A. Walther, L. Böhnisch \& K. Lenz (Hrsg.), Handbuch Übergänge (S. 651-687). Weinheim: Beltz Juventa.

Lenz, K., \& Schmitt, S. (2016). Anrechnung von außerhochschulisch erworbenen Kompetenzen als Instrument zur Öffnung von Hochschulen. In A. Wolter, U. Banscherus \& C. Kamm (Hrsg.), Zielgruppen Lebenslangen Lernens an Hochschulen (S. 321-343). Münster: Waxmann.

Lübben, S., Müskens, W., \& Zawacki-Richter, O. (2015). Nicht-traditionelle Studierende an deutschen Hochschulen. Implikationen unterschiedlicher Definitions- und Einteilungsansätze. In A. Hanft, O. Zawacki-Richter \& W.B. Gierke (Hrsg.), Herausforderung Heterogenität beim Übergang in die Hochschule (S. 29-51). Münster, New York: Waxmann.

Mangold, W. (1960). Gegenstand und Methode des Gruppendiskussionsverfahrens. Frankfurt a.M.: Europäische Verlagsanstalt.

Middendorff, E., Apolinarski, B., Becker, K., Bornkessel, P., Brandt, T., Heißenberg, S., \& Poskowsky, J. (2017). Die wirtschaftliche und soziale Lage der Studierenden in Deutschland 2016. 21. Sozialerhebung des Deutschen Studentenwerks durchgeführt vom Deutschen Zentrum für Hochschul- und Wissenschaftsforschung. http://www.sozialerhebung.de/download/21/Soz21_hauptbericht_barrierefrei. pdf. Zugegriffen: 07.06.2018.

Orr, D., \& Hovdhaugen, E. (2014). 'Second chance' routes into higher education: Sweden, Norway and Germany compared. International Journal of Lifelong Education, 33(1), 45-61.

Otto, A., \& Kamm, C. (2016). „Ich wollte einfach noch eine Stufe mehr“. Vorakademische Werdegänge und Studienentscheidungen von nicht-traditionellen Studierenden und ihr Übergang in der Hochschule. In A. Wolter, U. Banscherus \& C. Kamm (Hrsg.), Zielgruppen Lebenslangen Lernens an Hochschulen (S. 197-223). Münster: Waxmann. 
Rat der EU (2012). Empfehlungen des Rates der Europäischen Union vom 20. Dezember 2012 zur Validierung nichtformalen und informellen Lernens. (2012/C 398/01). https://eur-lex.europa.eu/legalcontent/DE/TXT/PDF/?uri=CELEX:32012H1222(01)\&from=EN. Zugegriffen: 07.06.2018.

Schäffter, O., \& Schicke, H. (2016). Anerkennung als Grundlage der Validierung. Reflexionen im Anschluss an eine „Pädagogik der Anerkennung“. DIE Zeitschrift für Erwachsenenbildung, 4, 26-30.

Schlögl, P. (2017). Schaffung eines strategischen Rahmens zur Validierung nicht formaler und informeller Lernergebnisse. Eine Zwischenbilanz aus Österreich. Berufsbildung in Wissenschaft und Praxis BWP, 46(6), 28-31.

Schreiber-Barsch, S. (2014). Sustainability and widening access to adult learners in higher education. REPORT Zeitschrift für Weiterbildungsforschung, 37(4), 41-53.

Slowey, M., \& Schuetze, H. G. (2012). All change-no change? In M. Slowey \& H. G. Schuetze (Hrsg.), Global perspectives on higher education and lifelong learners (S. 19-49). New York: Routledge.

Strauss, A., \& Corbin, J. (1996). Basics of qualitative research-techniques and procedures for developing grounded theory. London: SAGE.

Strübing, J. (2014). Grounded Theory. Zur sozialtheoretischen und epistemologischen Fundierung eines pragmatistischen Forschungsstils (3. Aufl.). Wiesbaden: Springer VS.

Struck, O. (2001). Gatekeeping zwischen Individuum, Organisation und Institution. Zur Bedeutung und Analyse von Gatekeeping am Beispiel von Übergängen im Lebensverlauf. In L. Leisering, R. Müller \& K. F. Schumann (Hrsg.), Institutionen und Lebensläufe im Wandel. Institutionelle Regulierungen von Lebensläufen (S. 29-54). Weinheim, München: Juventa.

Truschkat, I. (2013). Biografie und Übergang. In W. Schröer, B. Stauber, A. Walther, L. Böhnisch \& K. Lens (Hrsg.), Handbuch Übergänge (S. 44-63). Weinheim, Basel: Beltz Juventa.

UHH (2014). Service für Studierende. Campuscenter. Informationen zum Besonderen Hochschulzugang für Berufstätige nach § 38 des Hamburgischen Hochschulgesetzes. Stand: 13.02.2014. Hamburg: Universität Hamburg.

UHH (2017). Referat 31. Handreichung Nr. 14. Hochschulzugang für beruflich Qualifizierte ohne schulische HZB. Stand: 01/2017. Hamburg: Universität Hamburg.

Walther, A. (2015). Übergänge im Lebenslauf: Erziehungswissenschaftliche Heuristik oder pädagogische Gestaltungsaufgabe? In S. Schmidt-Lauff, H. von Felden \& H. Pätzold (Hrsg.), Transitionen in der Erwachsenenbildung. Gesellschaftliche, institutionelle und individuelle Übergänge (S. 35-56). Opladen, Berlin, Toronto: Barbara Budrich.

Walther, A., \& Stauber, B. (2013). Übergänge im Lebenslauf. In W. Schröer, B. Stauber, A. Walther, L. Böhnisch \& K. Lens (Hrsg.), Handbuch Übergänge (S. 23-43). Weinheim, Basel: Beltz Juventa.

Watson, L., Hagel, P., \& Chesters, J. (2013). A half-open door: pathways for VET award holders into Australian universities. Adelaide: NCVER Research Report.

Wolter, A., Banscherus, U., \& Kamm, C. (Hrsg.). (2016). Zielgruppen Lebenslangen Lernens an Hochschulen. Münster: Waxmann. 